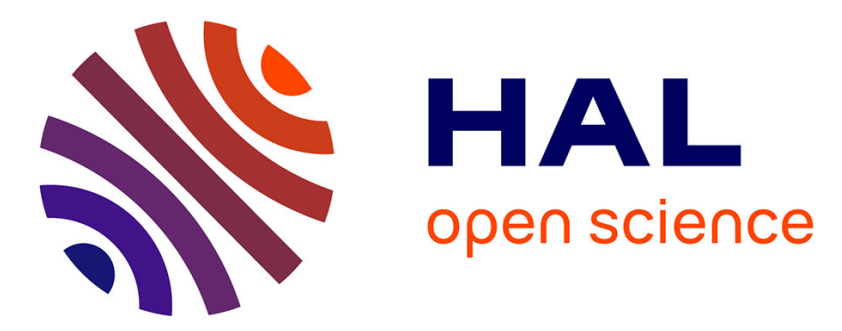

\title{
Regional Dynamic Traffic Assignment Framework for Macroscopic Fundamental Diagram Multi-regions Models
}

Sergio Batista, Ludovic Leclercq

\section{- To cite this version:}

Sergio Batista, Ludovic Leclercq. Regional Dynamic Traffic Assignment Framework for Macroscopic Fundamental Diagram Multi-regions Models. Transportation Science, 2019, 53 (6), pp1-28. 10.1287/trsc.2019.0921 . hal-02497177v2

\section{HAL Id: hal-02497177 \\ https://hal.science/hal-02497177v2}

Submitted on 24 Mar 2020

HAL is a multi-disciplinary open access archive for the deposit and dissemination of scientific research documents, whether they are published or not. The documents may come from teaching and research institutions in France or abroad, or from public or private research centers.
L'archive ouverte pluridisciplinaire HAL, est destinée au dépôt et à la diffusion de documents scientifiques de niveau recherche, publiés ou non, émanant des établissements d'enseignement et de recherche français ou étrangers, des laboratoires publics ou privés. 


\title{
Submitted to Transportation Science manuscript TS-2018-0395
}

Authors are encouraged to submit new papers to INFORMS journals by means of a style file template, which includes the journal title. However, use of a template does not certify that the paper has been accepted for publication in the named journal. INFORMS journal templates are for the exclusive purpose of submitting to an INFORMS journal and should not be used to distribute the papers in print or online or to submit the papers to another publication.

\section{Regional dynamic traffic assignment framework for MFD multi-regions models}

\author{
S. F. A. Batista \\ Univ. Lyon, ENTPE, IFSTTAR, LICIT, F-69518, Lyon, France, sergio.batista@ifsttar.fr \\ Ludovic Leclercq \\ Univ. Lyon, ENTPE, IFSTTAR, LICIT, F-69518, Lyon, France, ludovic.leclercq@entpe.fr
}

\begin{abstract}
In this paper, we propose a regional dynamic traffic assignment framework for Macroscopic Fundamental Diagram (MFD) models that explicitly accounts for trip length distributions. The proposed framework considers stochasticity on both the trip lengths and the regional mean speed. Consequently, we can define utility functions to assess the cost on alternatives, depending on which terms are considered stochastic. We propose a numerical resolution scheme based on Monte Carlo simulations and the Method of Successive Averages is used to solve the network equilibrium. Based on our test scenarios, we show that the variability of trip lengths inside the regions cannot be neglected. Moreover, it is also important to consider the stochasticity on the regional mean speeds to account for correlation between regional paths. The proposed regional dynamic traffic assignment is an extension of that discussed by Yildirimoglu and Geroliminis (2014). We also discuss an implementation of the proposed dynamic traffic assignment framework on the $6^{\text {th }}$ district of the Lyon network, where trip lengths are explicitly calculated. The traffic states are modeled by considering the accumulation-based MFD model. The results highlight the influence of the variability of trip lengths on the predicted traffic states.
\end{abstract}

Key words: Dynamic traffic assignment; Distributions of trip lengths; Macroscopic Fundamental Diagram;

Regional network; Regional paths

History: $15^{\text {th }}$ of May 2019

\section{Highlights}

- We discuss a regional dynamic traffic assignment framework for MFD-based models.

- The proposed framework explicitly accounts for trip length distributions and the evolution of traffic states in a regional network.

- This framework is able to capture the correlation between regional paths. 
- We show the importance of considering the combined effect of trip lengths and the evolution of traffic states on the network equilibrium calculation.

\section{Introduction}

Aggregated traffic models were first introduced by Godfrey (1969), Herman and Prigogine (1979) and Mahmassani, Williams, and Herman (1984). This approach was later revisited by the seminal works of Daganzo (2007) and Geroliminis and Daganzo (2008). The concept relies on partitioning the city network into a set of regions and then to represent flow exchanges. In Fig. 1 (a), we show an example of a city network. To divide the city network into regions, clustering techniques discussed in the literature can be used (e.g. Saeedmanesh and Geroliminis 2016, Lopez et al. 2017, Saeedmanesh and Geroliminis 2017, Casadei et al. 2018, Ambühl et al. 2019). The city network has to be transformed into a regional network, as shown in Fig. 1 (b) and Fig. 1 (c), to define routing options inside the regions. Let $X$ be the set of regions. Inside each region, the traffic conditions are characterized by a well-defined Macroscopic Fundamental Diagram (MFD). An MFD is a relationship between the average circulating flow $q_{r}([\mathrm{veh} / \mathrm{s}])$ and the accumulation $n_{r}([\mathrm{veh}])$ inside region $r$. The existence of this relationship was initially proved by Geroliminis and Daganzo (2008) using experimental data from Yokohama city (in Japan) and later confirmed by other authors (Geroliminis and Sun 2011, Ambühl and Menendez 2016, Loder et al. 2017). The traffic dynamics is defined by the following conservation equation for one region $r$ :

$$
\frac{d n_{r}(t)}{d t}=Q_{i n, r}(t)-Q_{o u t, r}(t), t>0
$$

where $n_{r}(t)$ is the accumulation of vehicles inside region $r$ at a given instant $t ; Q_{i n, r}(t)$ and $Q_{\text {out }, r}(t)$ are the inflow and outflow functions, respectively.

Depending on the assumptions made on the outflow function $Q_{o u t, r}$, two MFD-based models can be distinguished in the literature: the accumulation-based model (Daganzo 2007, Geroliminis and Daganzo 2008); and the trip-based model (Arnott 2013, Fosgerau 2015, Lamotte and Geroliminis 2016, Mariotte, Leclercq, and Laval 2017, Leclercq, Sénécat, and Mariotte 2017, Mariotte and Leclercq 2019).

The definition of a regional network (see Fig. 1 (b)) brings new challenges to build a dynamic traffic assignment framework for MFD-based models. The latter is related to the scaling of a city into a regional network and the definition of paths to define routing options. The city network (Fig. 1 (a)) includes all connected links corresponding to the existing roads. Local trips are defined by the sequence of links from the origin (o) to the destination (d) nodes inside the city network. The regional network is shown in Fig. 1 (b). A regional path is defined as the ordered sequence of 
(a)

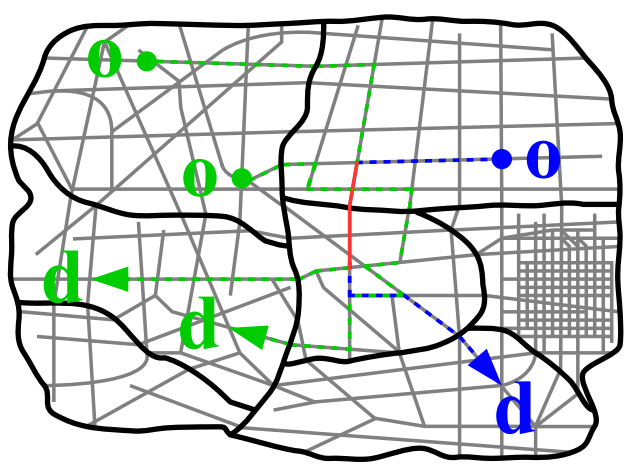

(c) (b)

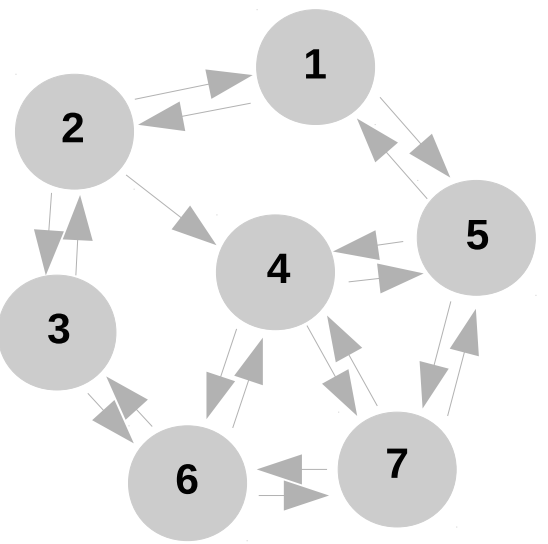

(d)

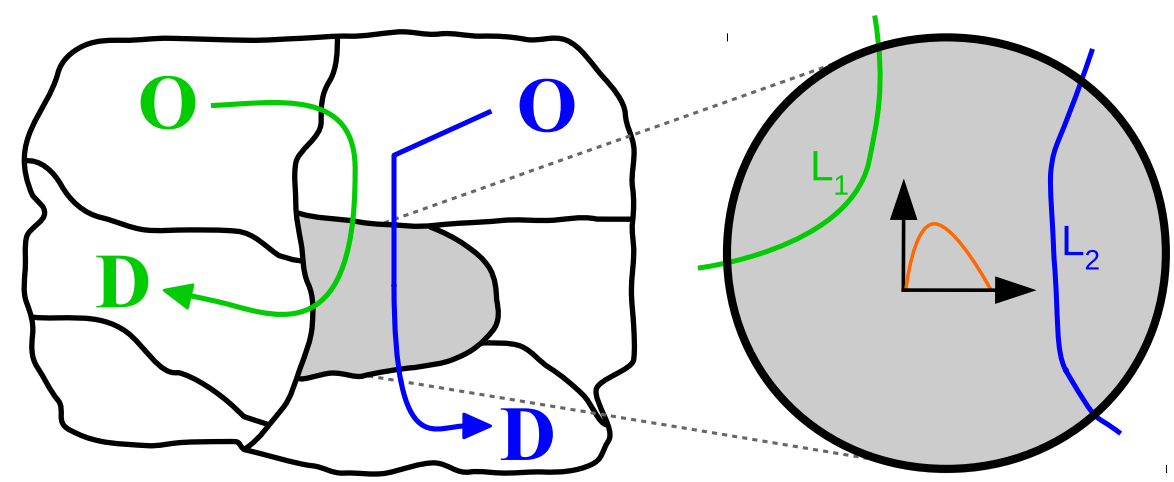

Figure 1 (a) City network with three trips represented. The partition of the city network is also shown. (b) Regional network where the gray circles represent the regions and the gray arrows represent the connections between the different regions. (c) Partition of the city network with the respective green and blue regional paths associated with the three trips. (d) Zoom of the gray region with a well-defined MFD function and crossed by the green and blue regional paths, each with a corresponding trip length $L_{1}$ and $L_{2}$.

regions that are crossed from the regional Origin (O) to Destination (D). Generically, a regional path $p$ can be defined as:

$$
p=\left(p_{1}, \ldots, p_{m}, \ldots, p_{R}\right), \forall m=1, \ldots, R \wedge m \in X
$$

where $R$ is the number of regions that define $p ; p_{1}$ and $p_{R}$ are the Origin $(\mathrm{O})$ and Destination (D) regions, respectively.

The literature on dynamic traffic assignment applied to regional networks and MFD based models is recent and not yet extensive. The first efforts to combine a dynamic traffic assignment framework with an MFD model were discussed by Leclercq and Geroliminis (2013) in the simplest case of 
parallel networks without path overlapping. Laval, Leclercq, and Chiabaut (2017) analyzed the analytical solutions of the Dynamic driver Equilibrium for a network composed of one OriginDestination and two alternatives: one freeway with a fixed capacity; and a city street modeled by MFD dynamics. For this simple network, the authors were able to determine analytical solutions for a non-overlapping network with constant trip lengths. Yildirimoglu and Geroliminis (2014) were certainly the first to propose a framework that can be applied to multi-regional MFD models. However, their framework did not have an explicit description of trip lengths in the regional network as trip lengths were updated at each iteration of the network loading. In Fig. 1 (a), we show three examples of trips highlighted in green and blue. The corresponding regional paths are shown in Fig. 1 (c). As highlighted by the green trips, several trips may exist in the city network that cross the same sequence of regions and consequently define the same regional path. The latter gives an interesting property to the regional paths. As can be observed in Fig. 1 (a), the green trips travel different distances inside each region they cross. The green regional path is then characterized by distributions of trip lengths inside each region. Let $L_{r p}$ be the set of trip lengths of regional path $p$ $\left(\forall p \in \Omega^{O D} \wedge \forall(O, D) \in W\right)$ inside region $\mathrm{r}(\forall r \in p)$. $\Omega^{O D}$ is the regional choice set for the OD pair and $W$ is the set of all OD pairs of the regional network. Batista et al. (2018) and Batista, Leclercq, and Geroliminis (2019) discuss several methods to characterize the trip length distributions of each regional path for a region, given the local trips in the city network as well as its partitioning. The methods differ from each other as a function of the level of aggregation of the trip length information inside regions, when scaling up the city into the regional network. As discussed in Batista et al. (2018) and Batista, Leclercq, and Geroliminis (2019), a similar distribution of trip lengths can be considered for all the regional paths that cross the same region. However, the description of regional trip lengths can be refined by considering the trip length of a regional path inside a region and taking into account the successive and/or previous regions, or even the specific regional path itself. In this paper, we will consider only the most detailed description, i.e. all regional paths have a specific trip length distribution inside each region.

An important aspect in dynamic network loading models is the correlation between paths. This dictates the sharing of information between different paths and how the path choices of different drivers will affect each other. In the city network, two trips are correlated if they share links in common (see for example the red links in Fig. 1 (a) that are shared by green and blue trips). The correlation between regional paths is different. To better emphasize this, we zoom on the gray region crossed by the blue and green regional paths (Fig. 1 (d)). The traffic states inside this region are described by a single MFD, e.g. in Fig. 1 (d). The correlation between the blue and green regional paths occurs due to the MFD assumption of homogeneous traffic conditions inside the gray region, i.e. all vehicles traveling inside this region are assumed to have the same 
speed. In fact, due to the MFD dynamics, a vehicle that enters the gray region will increase its accumulation $n_{r}$. This will automatically affect the region speed $v_{r}\left(n_{r}\right)$ and the travel times of all the vehicles already traveling inside this region, whatever their regional paths are. The dynamic traffic assignment framework discussed by Yildirimoglu and Geroliminis (2014) is based on the Multinomial Logit that does not account for correlations between regional paths.

In short, two important variables influence vehicles' travel times in the regional network: the trip length distributions of regional paths and the evolution of mean speed inside each region. The first element stems from the spatial distribution of local trips that are gathered to form a single regional path inside each region. The second element is related to the temporal distribution of regional speed over the assignment period, i.e. the period of time when regional path flow distributions are maintained constant. In this paper, we formulate a dynamic traffic assignment framework for MFD models that (i) is based on the explicit description of regional trip length distributions and (ii) accounts for the correlation between regional paths and traffic dynamics inside the regions. For this, we define different utility functions. In practice, only the more detailed utility function accounts for both factors simultaneously. The other definitions are set for the purposes of comparison and precisely assess the influence of these two factors. Network equilibrium is calculated through the Method of Successive Averages. Monte Carlo simulations are used to accommodate the regional path trip length distributions and the regional mean speed evolution over time. The methodology is described in more detail in Sect. 2. In Sect. 3, two simple test cases with one and two regions are presented to analyze in detail the components of the utility function definitions and to assess the importance of considering trip length and mean speed distributions. In Sect. 4, we then investigate the application of the proposed regional dynamic traffic assignment framework on the $6^{\text {th }}$ district of the Lyon network (France), where the trip lengths are explicitly scaled from the city network in accordance with the methodology discussed in Batista et al. (2018) and Batista, Leclercq, and Geroliminis (2019). In Sect. 5, we outline the conclusions of this paper.

\section{Regional dynamic traffic assignment: methodological framework}

In this section, we describe the methodological framework of the dynamic traffic assignment for multi-regional MFD models. In Table 1 we summarize the notations of all the symbols and variables used in this paper.

Table 1: Nomenclature used in this paper.

City network:

$o$

$d$
Origin node of a local trip in the city network. Destination node of a local trip in the city network. 
Table 1 - Continued from previous page

\begin{tabular}{|c|c|}
\hline$k$ & Local trip in the city network. \\
\hline$\Omega^{o d}$ & Route choice set of od pair. \\
\hline$\Xi$ & Set of all od pairs. \\
\hline$U_{k}^{o d}$ & Perceived travel time of local trip $k$ connecting the od pair. \\
\hline$T C_{k}^{o d}$ & Monetary cost associated with local trip $k$. \\
\hline$\beta^{o d}$ & Value of Time. \\
\hline$T T_{k}^{o d}$ & Travel time of trip $k$. \\
\hline$\overline{T T}_{k}^{o d}$ & Average travel time of local trip $k$. \\
\hline$\epsilon_{k}^{o d}$ & Error term that accounts for the trip travel time uncertainty. \\
\hline$\theta^{\text {od }}$ & Scale parameter of the Multinomial Logit model. \\
\hline \multicolumn{2}{|c|}{ Regional network: } \\
\hline$r$ & Region $r$. \\
\hline$O$ & Origin region of a regional path. \\
\hline$D$ & Destination region of a regional path. \\
\hline$X$ & Set of regions that define the regional network. \\
\hline$p$ & Regional path $p$. \\
\hline$R$ & Number of regions that define are regional path. \\
\hline$W$ & Set of all regional OD pairs. \\
\hline$\Psi$ & Set of all regional paths. \\
\hline$\Omega^{O D}$ & Regional choice set of regional OD pair. \\
\hline$T T_{p}^{O D}$ & Travel time of regional path $p$. \\
\hline$Q^{O D}$ & Total demand for OD pair. \\
\hline$\delta_{r p}$ & $\begin{array}{l}\text { Binary variable that equals } 1 \text { if regional path } p \text { crosses region } r \text {, or } 0 \\
\text { otherwise. }\end{array}$ \\
\hline$L_{r p}$ & Distribution of trip lengths of regional path $p$ inside region $r$. \\
\hline$v_{r}\left(n_{r}\right)$ & Speed-MFD of region $r$. \\
\hline$U_{p}^{O D}$ & Perceived cost of regional path $p$. \\
\hline$\sigma_{L}$ & Standard deviation of the $L_{r p}$ distribution. \\
\hline$\sigma_{v}$ & Standard deviation of $v_{r}\left(n_{r}\right)$ distribution. \\
\hline \multicolumn{2}{|c|}{ MFD-based models: } \\
\hline$t$ & Time instant. \\
\hline$q_{r}$ & Average circulation flow inside region $r$ in $[\mathrm{veh} / \mathrm{s}]$. \\
\hline$n_{r}$ & Accumulation of vehicles inside region $r$ in [veh]. \\
\hline$Q_{i n, r}(t)$ & Inflow function. \\
\hline$Q_{\text {out }, r}(t)$ & Outflow function. \\
\hline$n_{j a m}$ & Jam accumulation of the MFD in [veh]. \\
\hline$P_{\text {critical }}$ & Critical production of the MFD in [veh.m/s]. \\
\hline$u$ & Free-flow speed in $[\mathrm{m} / \mathrm{s}]$. \\
\hline \multicolumn{2}{|c|}{ Other variables and Method of Successive Averages: } \\
\hline$j$ & Iteration $j$ of the Method of Successive Averages. \\
\hline & Descent step of the Method of Successive Averages. \\
\hline$Q_{p}^{O D, j+1}$ & Path flow of regional path $p$ at iteration $j+1$ \\
\hline$Q_{p}^{O D, j}$ & Path flow of regional path $p$ at iteration $j$. \\
\hline$Q_{p}^{O D, *}$ & New temporary path flow of regional path $p$. \\
\hline$L_{r p}^{p}$ & Trip length sample obtained from the set $\left\{L_{r p}\right\}$. \\
\hline & Mean speed sample obtained from the set $v_{r}\left(n_{r}\right)$. \\
\hline
\end{tabular}


Table 1 - Continued from previous page

\begin{tabular}{ll}
\hline$N_{L_{r p}}^{M C}$ & Total number of samples gathered from $\left\{L_{r p}\right\}$. \\
$N_{v_{r}}^{M C}$ & Total number of samples gathered from $v_{r}\left(n_{r}\right)$. \\
$t o l$ & Pre-defined tolerance for the Gap criterion. \\
$N(\lambda)$ & Number of violations. \\
$\Phi$ & Pre-defined threshold for the number of violations. \\
$\alpha$ & Relative differences between regional path utilities. \\
$N_{\max }$ & Maximum number of descent step iterations. \\
$T$ & Simulation period.
\end{tabular}

\subsection{Traffic assignment models on city networks: a literature review}

The goal of traffic assignment models is to reproduce the patterns of vehicles travels in the city network. The first question related to traffic assignment is the definition of the choice set of plausible trips, i.e. a set of routes that drivers might probably choose. Note that, a route or a trip in the city network is represented by a sequence of links, with a fixed physical length, from the origin to the destination nodes. Let $\Omega^{\text {od }}$ be this trip choice set for the origin-destination (od) pair in the city network and $\Xi$ be the set of all od pairs in the city network. In the literature, there are several models designed for calculating $\Omega^{\text {od }}$. To name a few examples, we have the K-shortest path algorithm (van der Zijpp and Catalano 2005), the link elimination (Azevedo et al. 1993), the link penalty (de la Barra, Perez, and Anez 1993), the labeling approach (Ben-Akiva et al. 1984), the branch-and-bound algorithm (Prato and Bekhor 2006), the simulation approach (Nielsen 2000, Nielsen, Daly, and Frederiksen 2002, Ramming 2002, Bierlaire and Frejinger 2005, Bliemer, Bovy, and Li 2007) or the Metropolis-Hastings algorithm (Flötteröd and Bierlaire 2013).

The next question is how to reproduce the drivers' choices for their trips in the city network. The first ideas of traffic assignment date back to the two principles of Wardrop (Wardrop 1952). The first principle of Wardrop states that drivers are selfish and aim to minimize their own trip travel times. This is often referred in the literature as the User Equilibrium or Deterministic User Equilibrium (DUE). The second principle of Wardrop, also referred to as the System Optimum (SO), states that drivers choose their trips such that the sum of all travel times correspond to the minimum of the system.

The User Equilibrium principle assumes that drivers are perfectly rational and fully information about all possible routes choices as well as their travel times. However, the traffic conditions in the city network change over time and the increase of the congestion level leads to uncertain travel times. Generally, drivers evaluate their trip choices based on the perceived utility $U_{k}^{o d}$ :

$$
U_{k}^{o d}=T C_{k}^{o d}+\beta^{o d} T T_{k}^{o d}, \forall k \in \Omega^{o d} \wedge \forall(o, d) \in \Xi
$$


where $T C_{k}^{o d}$ is the monetary cost associated trip $k$ for od pair; $\beta^{o d}$ is the Value of Time (e.g. Lu, Mahmassani, and Zhou 2008, Zhang, Mahmassani, and Lu 2013); and $T T_{k}^{o d}$ is the perceived travel time for trip $k$ for od pair. The monetary costs can be associated, for example, with tolls, fuel consumption or public transport tickets. The Value of Time is the marginal cost between the trip monetary cost $T C_{k}^{o d}$ and its travel time $T T_{k}^{o d}$. For the sake of simplicity and without loss of generality, in this paper we only consider the trip travel times, i.e. there are no moneraty costs $T C_{k}^{o d}$ associated with the drivers' choices and $\beta$ is set to 1 . We then re-write the trip utility $U_{k}^{o d}$ as:

$$
\begin{aligned}
U_{k}^{o d} & =T T_{k}^{o d} \\
& =\overline{T T}_{k}^{o d}+\epsilon_{k}^{o d}, \forall k \in \Omega^{o d} \wedge \forall(o, d) \in \Xi
\end{aligned}
$$

where $\overline{T T_{k}^{o d}}$ is the mean travel time for trip $k$ and od pair; and $\epsilon_{k}^{o d}$ represents the error term that accounts for the travel time uncertainty. Eq. 4 represents an extesion of the User Equilibrium. The city network is said to be under Stochastic User Equilibrium (SUE) conditions when no driver can reduce his/her own perceived travel time by unilaterally switching their trip choice. The mathematical formulation of the SUE was introduced by Daganzo and Sheffi (1977) and Daganzo (1982).

Random Utility models (McFadden 1978) aim to incorporate the uncertainty term $\epsilon_{k}^{o d}$ in the modeling of the users' trip choices. These uncertainty terms are unobserved and one needs to make prior assumptions about their statistical distributions. The simplest model is the Multinomial Logit model (MNL) (Dial 1971), where $\epsilon_{k}^{o d}$ are assumed to be independently and identically Gumbel distributed, with a scale paramter $\theta^{\text {od }}$. The advantage of this model is the closed form for the calculation of the choice probabilities. However, it has two strong limitations. First, the scale parameter $\theta^{\text {od }}$ is calibrated at the od level (Chen et al. 2012). This implies that the travel times of all local trips connecting the same od pair have similar variances. Second, the MNL lacks the ability to capture the correlation between overlapping trips, i.e. trips that share one or a sequence of links in common. One solution to the previous limitation is to include a disutility correction factor, called Commonality Factor, in the deterministic term of the trip utility (see Eq. 4). This factor measures the similarity between two trips. Examples of this extension of the MNL are the C-Logit (Cascetta et al. 1996, Cascetta 2001), the Path Size Logit (Ben-Akiva and Bierlaire 1999, Frejinger, Bierlaire, and Ben-Akiva 2009) and the Path Size Correction (Bovy, Bekhor, and Prato 2008) models. Other authors propose to use the Paired Combinatorial Logit (Chu 1989, Prashker and Bekhor 2000, Bekhor and Prashker 2001) or the Cross Nested Logit (Vovsha 1997, Prashker and Bekhor 1998), where the correlation is taken into account in the stochastic term of the trip utility (see Eq. 4). The Mixed or Hybrid Logit (Bolduc 1999, Bekhor, Ben-Akiva, and Ramming 
2002, Frejinger and Bierlaire 2007) is a more sophisticated model, where the trip travel times are assumed to be the result of a sum of a Gumbel distributed term and a normally distributed term with zero mean and unit variance. The Probit model (Daganzo and Sheffi 1977) considers that the trip travel times are normally distributed. It allows a flexible definition of the covariance matrix and therefore the model is able to capture the correlation between overlapping trips. Contrary to the MNL, the Probit model does not have a closed form for the calculation of the choice probabilities, requiring the integration of multi-normal variate distributions over all alternative trips listed in $\Omega^{o d}$. This largely increases the computational burden required for the implementation of the Probit model. One solution is to utilize Monte Carlo simulations (Sheffi 1985) and discretize the trip travel time distributions into several realizations and locally solve deterministic assignment problems. For each realization set, drivers are assigned to the trip with the minimal travel time, similarly to the User Equilibrium principle. The final choices correspond to the average of all local choices. Prato (2009) provides a comprehensive review about the Random Utility models in the trip choice context.

Survey data (e.g. Zhu and Levinson 2015) shows that drivers do not always choose trips with the minimal travel times. The behavior assumptions of the User Equilibrium are relaxed to account for bounded rational users (Simon 1957, 1966, Mahmassani and Chang 1987, Di et al. 2013, 2014, Batista, Zhao, and Leclercq 2018). The trip choices of drivers are driven by aspiration levels that represent a set of target variables that should be satisfied, i.e. drivers choose trips that have a perceived travel time inferior to their aspiration level. This behavior is coined as satisficing, which results from the concatenation of the words suffice and satisfy. In traffic assignment problems, the calibration of the aspiration levels are in general based on the indifference band (Mahmassani and Chang 1987) concept. Other studies have considered that drivers are regret-averse (Chorus 2014, $\mathrm{Li}$ and Huang 2016) and aim to minimize their own regret with respect to the unselected trips.

The first implementations of traffic assignment models in a dynamic context, i.e. dynamic traffic assignment, date back to the works of Merchant and Nemhauser (1978a) and Merchant and Nemhauser (1978b) and have significantly evolved since then as discussed in the review papers of Peeta and Ziliaskopoulos (2001) and Viti and Tampère (2010). In the literature, one can find two approaches to solve a dynamic traffic assignment problem. The analytical approach (e.g. Wie, Tobin, and Carey 2002, Nie and Zhang 2010, Iryo 2011, Corthout et al. 2012) is suitable to study the unicity and uniqueness properties of the city network equilibrium. The simulation approach (e.g. Ben-Akiva, Gao, and Wen 2012, Mahmassani, Saberi, and Zockaie 2013, Shafiei, Gu, and Saberi 2018) utilizes traffic simulators to determine time-dependent trip travel times that account for congestion, shock-waves and spillback effects. In this paper, we focus on this second approach. Drivers are assigned to their trips based on a quasi-static approximation as function of the trips' 
travel times. This means that the total simulation period is split into several time intervals. The city network equilibrium is then calculated for each time interval, i.e. the path flow distribution for od pair is constant during a time interval. We can adjust the length of these time intervals to update more frequently the path flows for cases when the demand suffers quick changes or when the traffic states are quickly evolving.

\subsection{Scale-up of city into regional networks: motivation for the regioanl DTA framework}

In this paper, we focus on the development of a network loading framework to solve the dynamic traffic assignment problems in regional networks. Fig. 2 depicts a flowchart that summarizes each step of the regional dynamic traffic assignment framework. It requires as an input the definition of the city network partitioning, i.e. the set $X$, and a set of trips in the city network. The city network can be partitioned into regions using any of the techniques described in the literature (Saeedmanesh and Geroliminis 2016, 2017, Lopez et al. 2017, Casadei et al. 2018, Ambühl et al. 2019). The challenging question is how to define the set of trips in the city network. The full trip patterns in a city network are unknown and change over time. One solution to address this issue is to construct a set of virtual trips (Batista and Leclercq 2018, Batista, Leclercq, and Geroliminis 2019). This is done by randomly sampling several origin-destination pairs in the city network and then calculate the shortest-path in distance for each one. Each virtual trip represent an individual travel in the city network. The scale-up of this virtual trips into paths in the regional network brings new challenges. The first question is how to define the regional paths and the regional choice set $\Omega^{O D}$. The second question is related with the characterization of the regional paths through distributions of trip lengths. The answers to these two questions make the difference between the regional and the classical city-network based dynamic traffic assignment frameworks.

In city networks, the trip choice set $\Omega^{\text {od }}$ is obtained either by shortest-paths calculations or by any other model described in the previous Sect. 2.1. Regional paths are the result of the scaling-up of virtual trips in the city network following the sequence of regions they cross according to the city network partitioning (Batista and Leclercq 2018). We define the frequency of a regional path by the number of virtual trips it has associated. For each OD pair, the regional path with the largest number of virtual trips associated is then the most frequent one. The regional choice set $\Omega^{O D}$ is composed by the three most frequent regional paths. We define $\Psi$ as the set of all regional paths.

The next step consists in calculating of the trip length distributions. Virtual trips in the city network are composed by a sequence of links that have a fixed length. However, the case of regional paths is more complex. As depicted in Fig. 1, the virtual trips that define the same regional path have different travel distances in the regions they cross. Batista, Leclercq, and Geroliminis (2019) propose a methodological framework to explicitly calculate trip length distributions given 
the virtual trips set and different levels of information from the regional network. The latter ranges from no information about the previous and next regions to be traveled by the virtual trips, to their associated regional path. The first level of information determinates trip length distributions based on all virtual trips that cross one region. Therefore, this distribution is similar to all regional paths that cross one region. The most detailed level of information only considers the associated virtual trips to one regional path to calculate the trip length distributions. Batista, Leclercq, and Geroliminis (2019) show that the first level of information is not able to capture the variability of the trip lengths of all regional paths crossing the same region. The authors also show that the calibration of trip lengths clearly influence the traffic dynamics in the regions. The authors conclude that filtering the virtual trips by their associated regional path to explicitly distributions of trip lengths should be considered.

In regional networks there are two factors that influence the travel times of the regional paths. The travel times evolve with the changes of the traffic conditions in each link of the city network. In regional networks, travel times are not only influenced by changes of the traffic conditions inside regions, but also by the trip length distributions. We notice here a clear difference with the city network, where link lengths are constant and similar for all paths that take the same link. Not only do the regional paths that cross the same region have different mean trip lengths, they also have different trip length distributions (Batista et al. 2018, Batista, Leclercq, and Geroliminis 2019, Batista and Leclercq 2018). Therefore, the travel time of a $p$ of regional OD pair, $T T_{p}^{O D}$, can be calculated as:

$$
T T_{p}^{O D}=\sum_{r \in X}\left(\frac{L_{r p}}{v_{r}\left(n_{r}\right)}\right) \delta_{r p}, \forall p \in \Omega^{O D} \wedge \forall(O, D) \in W
$$

where $\delta_{r p}$ is a binary variable that equals 1 if regional path $p$ crosses region $r$, or 0 otherwise.

\subsection{Formulation of the regional DTA framework and solution algorithm}

The question is how to calculate the regional network equilibrium given the evolution of the traffic conditions in the regions as well as the explicitly calculated distributions of trip lengths that characterize the regional paths. To answer this question, we consider that the following two terms can be distributed:

- the empirical set of trip lengths $\left\{L_{r p}\right\}$, for each region $\mathrm{r}$ that defines $p$.

- the time varying speed-MFD set $v_{r}\left(n_{r}\right)$ of each region $\mathrm{r}$ that defines $p$.

Depending on which distributions are considered, four utility functions can be defined to describe all regional path alternatives for each OD pair. In this paper, we target the User Equilibrium, considering different formulations of the utility function. They are defined based on a first order Taylor expansion of Eq. 5 around the mean values of $\bar{L}_{r p}$ and $\bar{v}_{r}$ as well as on which terms we consider to be distributed. Then, we define the four following utility functions: 


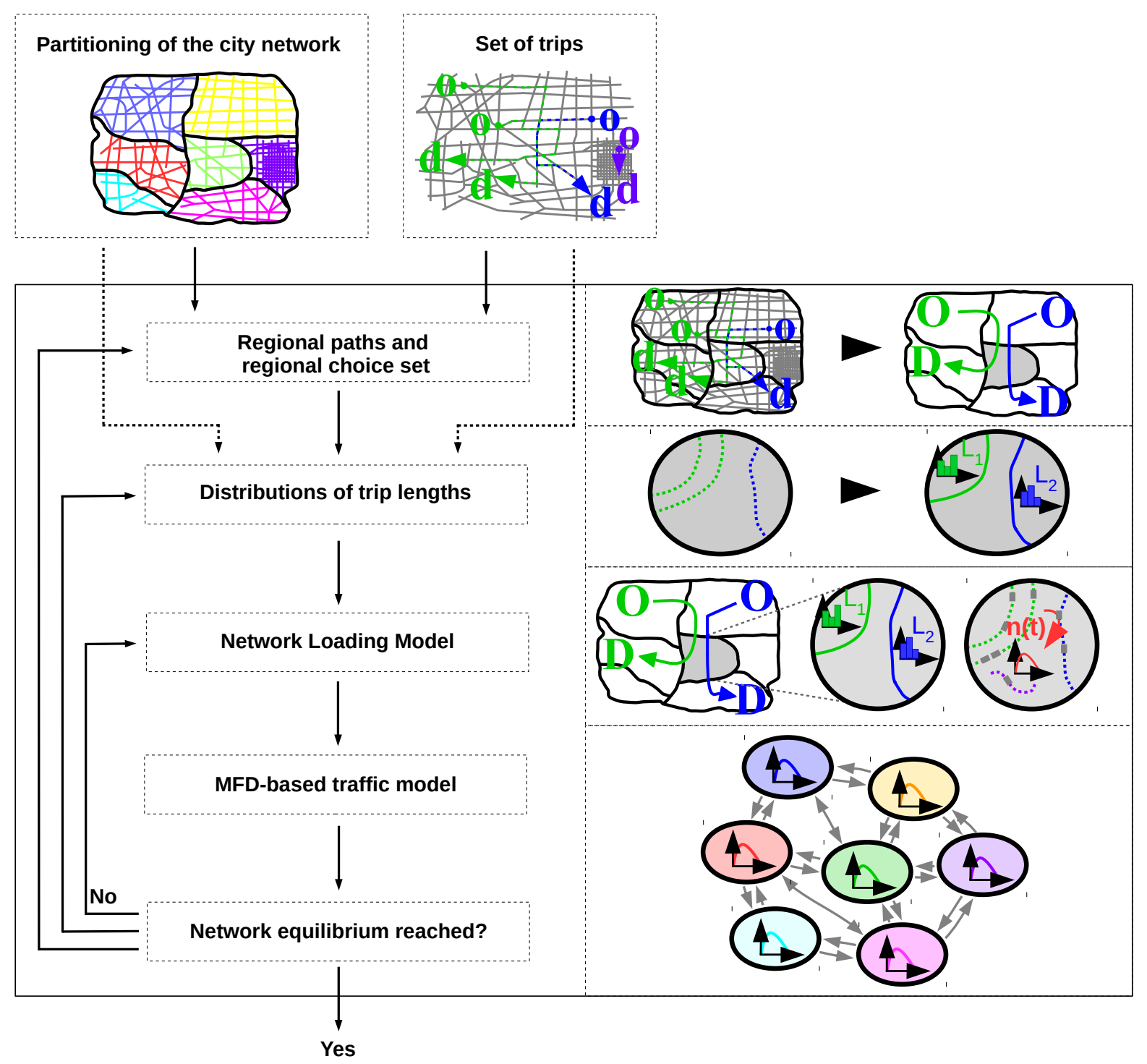

Figure 2 Flowchart that summarizes the different steps of the regional dynamic traffic assignment framework.

- Utility 1: neither the set of trip lengths $L_{r p}$ nor the set of mean speeds $v_{r}\left(n_{r}\right)$ are considered distributed. The perceived $\operatorname{cost} U_{p}^{O D}$ is:

$$
U_{p}^{O D}=\sum_{r \in X}\left(\frac{\bar{L}_{r p}}{\bar{v}_{r}}\right) \delta_{r p}, \forall p \in \Omega^{O D} \wedge \forall(O, D) \in W
$$

where $\bar{L}_{r p}$ is the average trip length of regional path $p$ inside region $r$; and $\bar{v}_{r}$ is the mean speed of region $r$. 
- Utility 2: only the set of trip lengths $L_{r p}$ is considered distributed. The perceived cost $U_{p}^{O D}$ is:

$$
\begin{aligned}
U_{p}^{O D} & =\sum_{r \in X}\left(\frac{\bar{L}_{r p}}{\bar{v}_{r}}+\frac{1}{\bar{v}_{r}}\left(L_{r p}-\bar{L}_{r p}\right)\right) \delta_{r p} \\
& =\sum_{r \in X}\left(\frac{L_{r p}}{\bar{v}_{r}}\right) \delta_{r p}, \forall p \in \Omega^{O D} \wedge \forall(O, D) \in W
\end{aligned}
$$

- Utility 3: only the set of mean speed $v_{r}\left(n_{r}\right)$ is considered distributed. The perceived cost $U_{p}^{O D}$ is:

$$
\begin{aligned}
U_{p}^{O D} & =\sum_{r \in X}\left(\frac{\bar{L}_{r p}}{\bar{v}_{r}}-\frac{\bar{L}_{r p}}{\bar{v}_{r}^{2}}\left(v_{r}-\bar{v}_{r}\right)\right) \delta_{r p} \\
& =\sum_{r \in X}\left(2 \frac{\bar{L}_{r p}}{\bar{v}_{r}}-\frac{\bar{L}_{r p} v_{r}}{\bar{v}_{r}^{2}}\right) \delta_{r p}, \forall p \in \Omega^{O D} \wedge \forall(O, D) \in W
\end{aligned}
$$

- Utility 4: both the sets of trip lengths $L_{r p}$ and mean speeds $v_{r}\left(n_{r}\right)$ are considered distributed. The perceived cost $U_{p}^{O D}$ is:

$$
\begin{aligned}
U_{p}^{O D} & =\sum_{r \in X}\left(\frac{\bar{L}_{r p}}{\bar{v}_{r}}+\frac{1}{\bar{v}_{r}}\left(L_{r p}-\bar{L}_{r p}\right)-\frac{\bar{L}_{r p}}{\bar{v}_{r}^{2}}\left(v_{r}-\bar{v}_{r}\right)\right) \delta_{r p} \\
& =\sum_{r \in X}\left(\frac{\bar{L}_{r p}}{\bar{v}_{r}}+\frac{L_{r p}}{\bar{v}_{r}}-\frac{\bar{L}_{r p} v_{r}}{\bar{v}_{r}^{2}}\right) \delta_{r p}, \forall p \in \Omega^{O D} \wedge \forall(O, D) \in W
\end{aligned}
$$

where $v_{r}$ is a conditional distribution of $L_{r p}$ during the simulation period.

These four utility function definitions, defined by Eq. 6 to Eq. 9, allow calculating different network equilibria. Hereafter, we refer to the network equilibrium related to the utility definitions from Eq. 6 to Eq. 9 as Equilibrium 1 to Equilibrium 4, respectively. In this paper, we consider Equilibrium 4 as the reference because it accounts for distributions of the two key elements mentioned in the introduction, i.e. $L_{r p}$ and $v_{r}\left(n_{r}\right)$.

To calculate the regional network equilibrium defined by any of the utility functions Eq. 6 to Eq. 9, we consider the Method of Successive Averages (MSA). The MSA is based on a descent step finding procedure. The new regional path flows $Q_{p}^{O D, j+1}$, at descent step $j+1$, are updated according to the regional path flows $Q_{p}^{O D, j}$, at descent step $j$, and to the new temporary regional path flows $Q_{p}^{O D, *}, \forall(O, D) \in W$, as:

$$
Q_{p}^{O D, j+1}=Q_{p}^{O D, j}+\eta_{j}\left\{Q_{p}^{O D, *}-Q_{p}^{O D, j}\right\}, \forall p \in \Omega^{O D} \wedge \forall(O, D) \in W
$$

The question is how to determine the new temporary regional path flows $Q_{p}^{O D, *}$, considering the utility functions defined in Eq. 6 to Eq. 9. Let $P_{p}^{O D}$ be the probability of choosing regional path 
$p$ from $\Omega^{O D}, \forall(O, D) \in W$. The regional path flows $Q_{p}^{O D, *}$ are calculated as follows (Daganzo and Sheffi 1977, Daganzo 1982):

$$
\begin{aligned}
Q_{p}^{O D, *} & =Q^{O D} P_{p}^{O D} \\
& =Q^{O D} P_{p}^{O D}\left(U_{p}^{O D} \leq U_{j}^{O D}\right), \forall j \neq p \wedge \forall(j, p) \in \Omega^{O D} \wedge \forall(O, D) \in W
\end{aligned}
$$

where $Q^{O D}$ is the total demand of OD pair; and $P_{p}^{O D}$ is the probability of choosing regional path $p$ from the regional choice set $\Omega^{O D}, \forall(O, D) \in W$.

The probability terms are calculated through Monte Carlo simulations. From the sets $\left\{L_{r p}\right\}$ and $v_{r}\left(n_{r}\right)$, we gather samples $L_{r p}^{l}$ and $v_{r}^{h}$, respectively, depending on the specificities of each utility function definition. Note that $v_{r}\left(n_{r}\right)$ time series is given considering the simulation time-step of 1 second. Moreover, $v_{r}\left(n_{r}\right)$ is a conditional distribution given the distribution of trip lengths $\left\{L_{r p}\right\}$ throughout the simulation period. The dynamic network loading is performed given trip length distributions for regional paths, directly influencing $v_{r}\left(n_{r}\right)$. For each Monte Carlo trial and each OD pair, we calculate the regional path utilities and assign drivers based on an all-or-nothing procedure to the regional path with the minimal utility. The new temporary regional path flows $Q_{p}^{O D, *}$ are updated by averaging all the drivers choices over all Monte Carlo samples. In more detail for each regional network equilibrium:

- Equilibrium 1: For each regional OD pair, we calculate the mean speed $\bar{v}_{r}$ and mean trip length $\bar{L}_{r p}$ and then the regional path costs following Eq. 6. The probability $P_{p}^{O D}=1$ when regional path $p$ has the minimal travel time for the OD pair. Then, $Q_{p}^{O D, *}=1, \forall p \in \Omega^{O D} \wedge \forall(O, D) \in W$.

- Equilibrium 2: For each regional path, we uniformly draw trip length samples $L_{r p}^{l}$ from $\left\{L_{r p}\right\}$. Let $N_{L_{r p}}^{M C}$ be the total number of samples considered for the trip lengths. We also calculate the mean speed $\bar{v}_{r}$ for each region. The trip length distribution $L_{r p}$ in Eq. 7 is discretized into the $L_{r p}^{l}$ samples. From Eq. 7, the regional path cost $U_{p}^{l}$ is calculated as:

$$
U_{p}^{l}=\sum_{r \in X}\left(\frac{L_{r p}^{l}}{\bar{v}_{r}}\right) \delta_{r p}, \forall p \in \Omega^{O D} \wedge \forall(O, D) \in W
$$

- Equilibrium 3: For each regional path, we uniformly draw mean speed samples $v_{r}^{h}$ from $v_{r}\left(n_{r}\right)$ time series. Let $N_{v_{r}}^{M C}$ be the total number of samples for the mean speed $v_{r}\left(n_{r}\right)$ set. We calculate the mean speed $\bar{v}_{r}$ for each region and the average trip lengths of each regional path $p$ inside region $r$. The mean speed distribution $v_{r}\left(n_{r}\right)$ in Eq. 8 is discretized into the $v_{r}^{h}$ samples. From Eq. 8, the regional path cost $U_{p}^{h}$ is calculated as:

$$
U_{p}^{h}=\sum_{r \in X}\left(2 \frac{\bar{L}_{r p}}{\bar{v}_{r}}-\frac{\bar{L}_{r p} v_{r}^{h}}{\bar{v}_{r}^{2}}\right) \delta_{r p}, \forall p \in \Omega^{O D} \wedge \forall(O, D) \in W
$$


- Equilibrium 4: For each regional path, we independently draw uniform trip length samples $L_{r p}^{l}$ from $\left\{L_{r p}\right\}$ and mean speed samples $v_{r}^{h}$ from $v_{r}\left(n_{r}\right)$. The mean speed $\bar{v}_{r}$ for each region and the average trip lengths of each regional path $p$ inside region $r$ are also calculated. In Eq. 9, both the $L_{r p}$ and $v_{r}\left(n_{r}\right)$ distributions are discretized into the $L_{r p}^{l}$ and $v_{r}^{h}$ samples, respectively. From Eq. 9, the regional path $\operatorname{cost} U_{p}^{l h}$ is calculated as:

$$
U_{p}^{l h}=\sum_{r \in X}\left(\frac{\bar{L}_{r p}}{\bar{v}_{r}}+\frac{L_{r p}^{l}}{\bar{v}_{r}}-\frac{\bar{L}_{r p} v_{r}^{h}}{\bar{v}_{r}^{2}}\right) \delta_{r p}, \forall p \in \Omega^{O D} \wedge \forall(O, D) \in W
$$

For the MSA implementation, we also have to define the descent step $\eta_{j}$, where $j$ is the descent iteration, to ensure good convergence properties. In this paper, we consider that $\eta_{j}=\frac{1}{j}$. On the other hand, we must also define the convergence criteria of the MSA algorithm. To do this, we follow the work of Sbayti, Lu, and Mahmassani (2007) and consider:

- the relative Gap:

$$
G a p=\frac{\sum_{O} \sum_{D} \sum_{p \in \Omega^{O D}} Q_{p}^{O D}\left(V_{p}^{O D}-\min \left(\overrightarrow{V^{O D}}\right)\right)}{\left.\sum_{O} \sum_{D} Q^{O D} \min \left(\overrightarrow{V^{O D}}\right)\right)}
$$

where $\overrightarrow{V^{O D}}=\left\{V_{p}^{O D}\right\}, \forall p \in \Omega^{O D} \wedge \forall(O, D) \in W$. The Gap function is also an indicator of the network equilibrium quality that indicates how far we are from the driver Equilibrium. We consider that the network equilibrium is achieved if Gap $\leq$ tol, where tol is a pre-defined tolerance.

- the number of violations $N(\lambda)$. It consists in comparing the regional path flows at descent iteration $j+1, Q_{p}^{O D, j+1}$, with those at descent iteration $j, Q_{p}^{O D, j} . N(\lambda)$ represents the number of cases where $\left|Q_{p}^{O D, j+1}-Q_{p}^{O D, j}\right|$ is higher than a pre-defined threshold $\Phi$ (Sbayti, Lu, and Mahmassani 2007), where $\Phi$ is an upper bound. Convergence is achieved if $N(\lambda) \leq \Phi$.

- a maximum number of iterations $N_{\max }$.

The MSA loop is repeated until the convergence criteria are satisfied. Algorithm 1 summarizes the MSA loop and Monte Carlo implementations for the four utility functions.

In the next sections, we investigate the relevance of considering both the empirical set of trip lengths $\left\{L_{r p}\right\}$ as well as the time-varying speed-MFD set $v_{r}\left(n_{r}\right)$ in the calculation of the regional network equilibrium.

\section{Analysis of the regional dynamic traffic assignment framework on simple regional networks}

In this section, we analyze the implementation of the regional dynamic traffic assignment framework, discussed in the previous section, in two simple regional networks: one region and one regional OD pair (Sect. 3.2); and two regions and two regional OD pairs (Sect. 3.3). In Sect. 3.4, we investigate if the proposed framework properly handles the correlation between regional paths. 


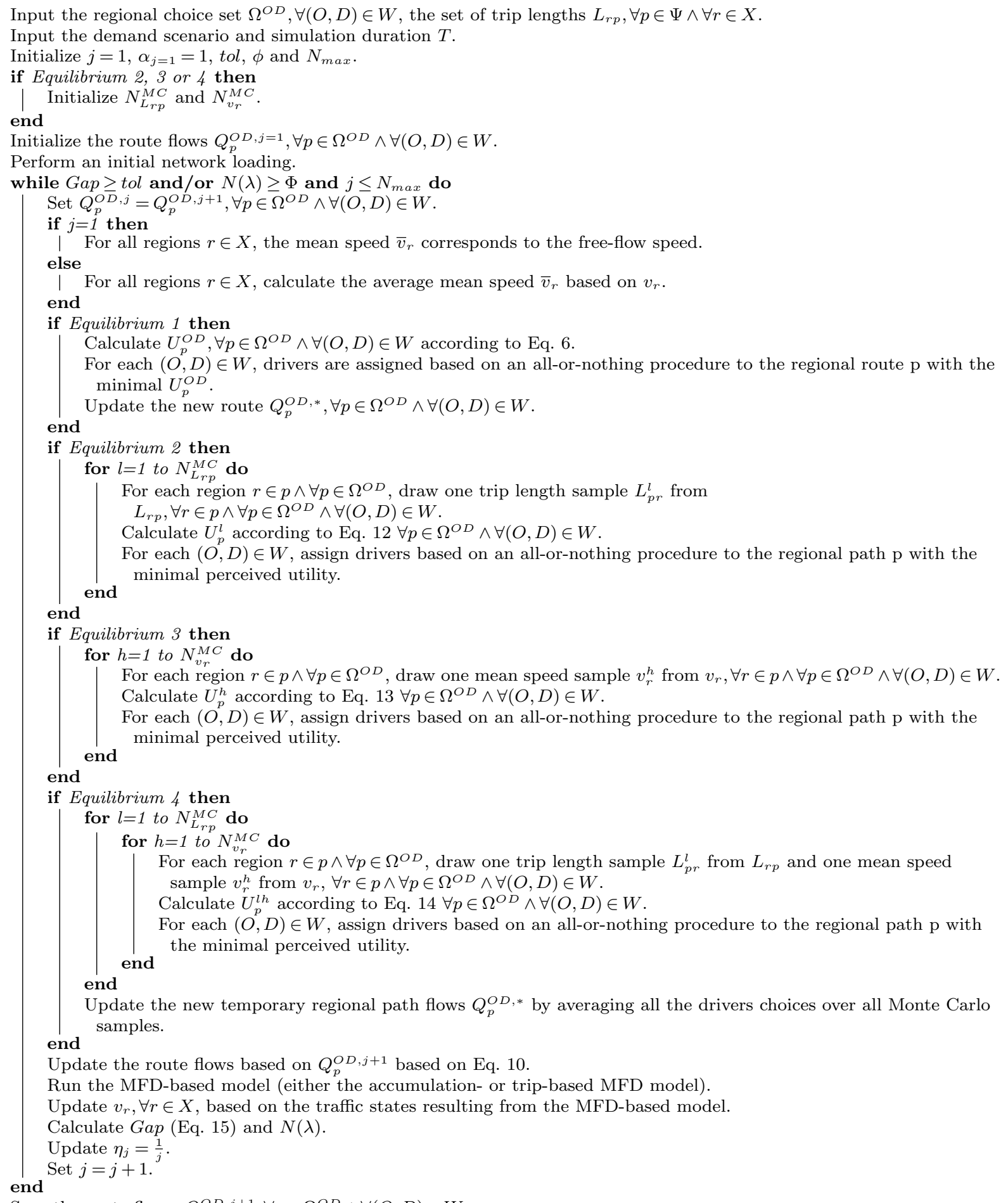

Save the route flows: $Q_{p}^{O D, j+1}, \forall p \in \Omega^{O D} \wedge \forall(O, D) \in W$.

Algorithm 1: Regional dynamic traffic assignment framework for MFD-based models.

\subsection{Test scenarios definition}

For the first test scenario, we consider a regional network consisting of one region and one regional OD pair connected by two regional paths. We show the regional network in Fig. 3, where the two 
regional paths are highlighted in orange. Let $L_{1}$ and $L_{2}$ be the distributions of trip lengths inside the region. We consider a bi-parabolic MFD function with: jam accumulation $n_{\text {jam }}=1000$ veh; critical production $P_{\text {critical }}=3000$ veh.m/s; and free-flow speed $u=15 \mathrm{~m} / \mathrm{s}$.

For the second test scenario, we consider a regional network consisting of two regions and two regional OD pairs connected by two regional paths each. We show the regional network in Fig. 4. Let: $L_{1}$ and $L_{2}$ be the regional trip length distributions for the orange regional paths; and $L_{3}$ and $L_{4}$ be the regional trip length distributions for the green regional paths. The traffic states inside each of the two regions are defined by a parabolic MFD function with: jam accumulation $n_{\text {jam }}=1000$ veh; critical production $P_{\text {critical }}=2000$ veh.m $/ \mathrm{s}$; and free-flow speed $u=15 \mathrm{~m} / \mathrm{s}$.

For all the MFD simulations performed in this section, we consider a simulation period of $T=800$ seconds. For the network equilibrium convergence, we set a maximum number of violations of $\Phi=0$ and/or a Gap tolerance $t o l \leq 0.01$ and a maximum of descent step iterations of $N_{\max }=100$. For the Monte Carlo simulations, we consider $N_{L_{r p}}^{M C}=N_{v_{r}}^{M C}=10000$ samples.
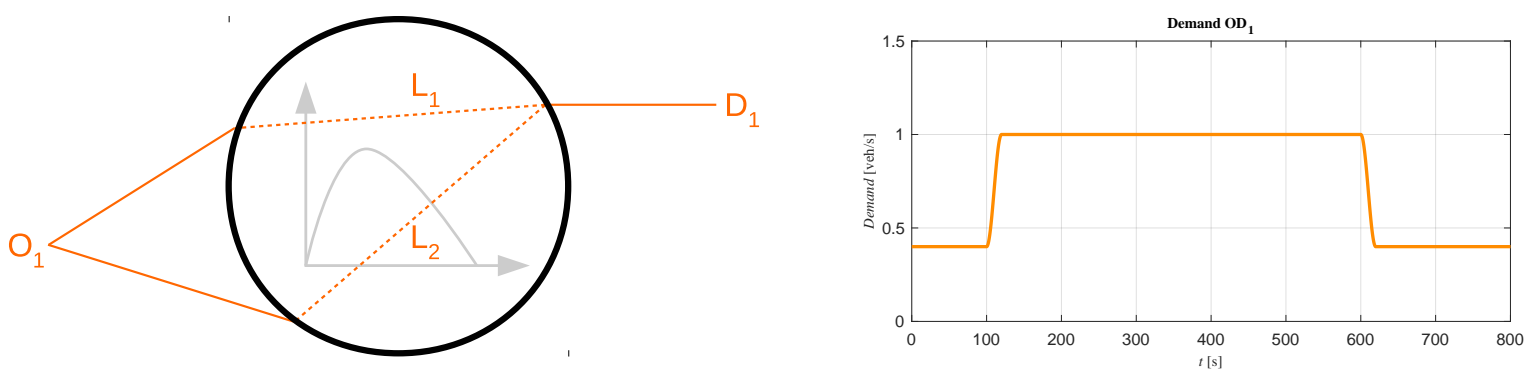

Figure 3 Left: Regional network. One regional OD pair is shown, with two regional paths with distributions of regional trip lengths $L_{1}$ and $L_{2}$. Right: The demand scenario for the OD pair and for the tests of Sect. 3.2 and Sect. 3.4 .

\subsection{Analysis of the regional path flows at equilibrium: 1-region test case}

We analyze the regional path flows calculated for the four network equilibrium definitions (i.e. defined Eq. 6 and Eq. 9). We investigate how different the regional path flows for the reference Equilibrium 4 are in comparison to the other three network equilibria. The trip lengths for the two regional paths are sampled according to a normal distribution with: a fixed mean $\bar{L}_{2}=1500[\mathrm{~m}]$ for regional path 2; and a varying mean values between 1300 to 1700 [m] increasing step size by 25 $[\mathrm{m}]$ for regional path 1 . We consider three values of the standard deviation $\sigma_{L}=50,100,200[\mathrm{~m}]$, similar for both regional paths.

In Fig. 5, we show the regional path flows for the four network equilibria as a function of $\bar{L}_{1}$ and the three values of $\sigma_{L}$. 

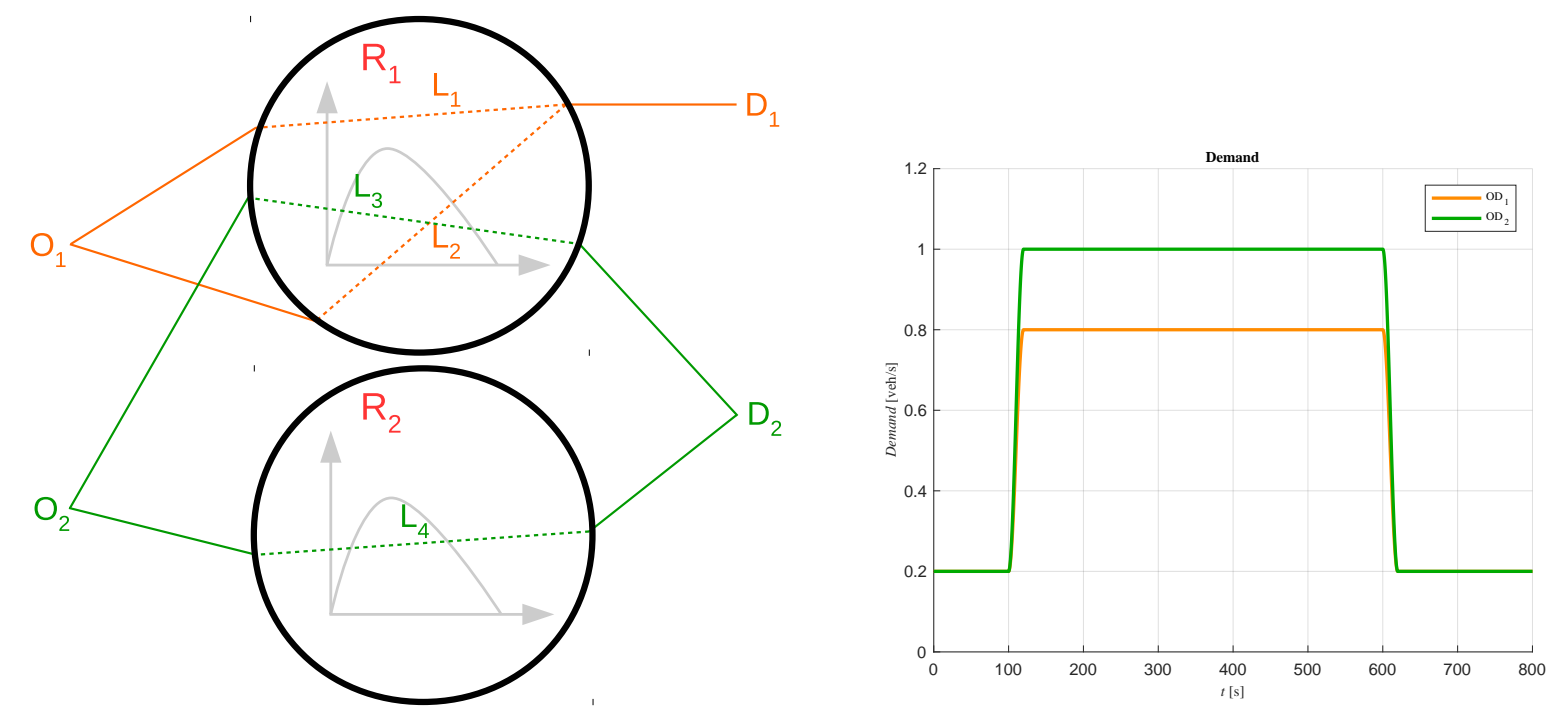

Figure 4 Left: Regional network consisting of two regions labeled $R_{1}$ and $R_{2}$. Two DO pairs are shown with two regional paths connecting each one. Right: Demand scenario for the two OD pairs and for the tests of Sect. 3.3 and Sect. 3.4.

Equilibrium 1 is purely deterministic since we consider that neither the $L_{r p}$ nor $v_{r}$ are distributed. For this equilibrium, since the MFD dynamics is the same for both regional paths, only the average trip lengths $\bar{L}_{1}$ and $\bar{L}_{2}$ modify the perceived utility functions. When $\bar{L}_{1}<\bar{L}_{2}$, implying $U_{1}<U_{2}$, all vehicles choose the minimum utility that corresponds to regional path 1 . For $\bar{L}_{1}=\bar{L}_{2}=1500[\mathrm{~m}]$, implying $U_{1}=U_{2}$, vehicles equally choose regional paths 1 and 2. For $\bar{L}_{1}>\bar{L}_{2}$, implying $U_{1}>U_{2}$, all vehicles choose regional path 2 . The evolution of the regional path flows as a function of the increase of $\bar{L}_{1}$ is completely different to those obtained by our reference, i.e. Equilibrium 4.

For Equilibrium 3, the regional path flows at equilibrium depend on the average trip lengths $\bar{L}_{1}$ and $\bar{L}_{2}$ and on the mean speed distribution $v_{r}\left(n_{r}\right)$ (see Eq. 8). This definition of the network equilibrium is independent of $\sigma_{L}$. Consequently, only one evolution of the regional path flows is shown by the dashed black line in Fig. 5. In Fig. 6, we show the evolution of the speed $v_{r}[\mathrm{~m} / \mathrm{s}]$ for Equilibrium 3 and four values of $\bar{L}_{1}[\mathrm{~m}]$. In Fig. 7, we show the evolution of the mean speed $\bar{v}_{1}$ and of the standard deviation $\sigma_{v_{1}}$ as a function of the $\bar{L}_{1}$ increase. In Fig. 8, we show the distributions of the regional path utilities $U_{1}^{O D_{1}}$ and $U_{2}^{O D_{1}}$. We also set a criterion $\alpha$ that defines the normalized difference between the regional path utilities:

$$
\alpha=\frac{U_{2}^{O D_{1}}-U_{1}^{O D_{1}}}{U_{2}^{O D_{1}}}
$$

The $\alpha$ criterion allows predicting the route flow distributions. If $\alpha>0$, the vehicles choose regional path 2 for these Monte Carlo trials. The distributions of $\alpha$ directly gives an immediate overview of the flow distribution between the two regional paths. The distributions of the $\alpha$ criterion are 

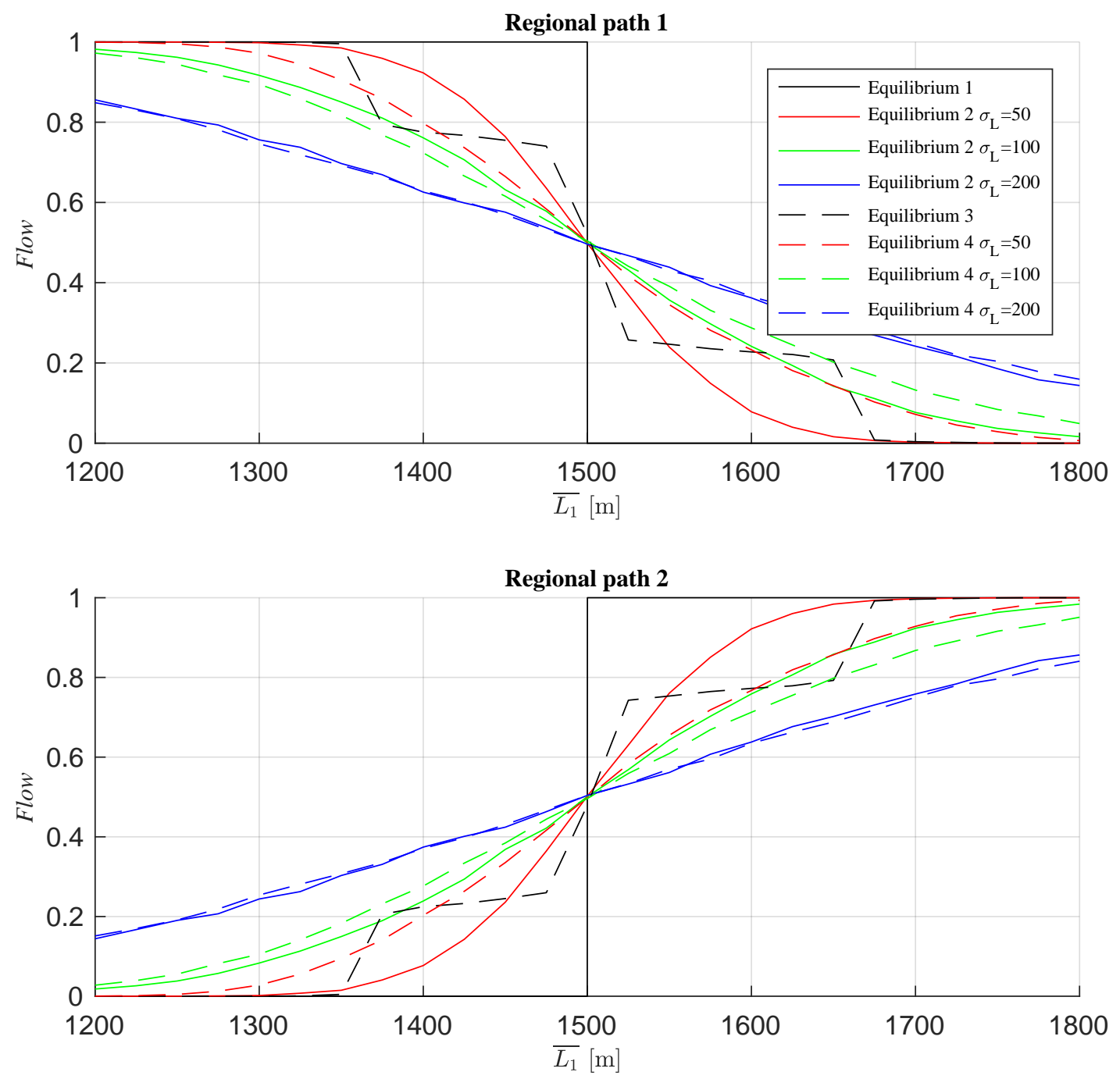

Figure 5 Regional path flows for the four network equilibria as function of $\bar{L}_{1}$ and for the 1 region network (Fig. 3). For Equilibrium 2 and 4, three values of $\sigma_{L}$ are considered.

also shown in Fig. 8, for four values of $\bar{L}_{1}$ [m]. In Fig. 8, we observe the presence of two peaks for the regional path utilities $U_{1}^{O D_{1}}$ and $U_{2}^{O D_{1}}$. These peaks correspond to the fluid and congested regimes of the traffic states inside the region. This is also observed in the evolution of the speed $v_{1}$ in Fig. 6. In Fig. 7, we observe that both the mean speed $\bar{v}_{1}$ and the standard deviation $\sigma_{v_{1}}$ do not vary greatly as $\bar{L}_{1}$ increases. Moreover, $\sigma_{v_{1}}$ is small. The regional path flows at equilibrium will then be more dependent on how close the average trip lengths are $\bar{L}_{1}$ and $\bar{L}_{2}$. We depict this in more detail, analyzing the regional path utility distributions shown in Fig. 8. For $\bar{L}_{1}=1300$ $[\mathrm{m}], \sigma_{v_{1}}$ is not sufficiently large to compensate the distance between $\bar{L}_{1}$ and $\bar{L}_{2}$. From the Monte Carlo trials, we obtain $U_{2}^{O D_{1}}>U_{1}^{O D_{1}}$ for all the samples, as shown by the $\alpha$ distribution in Fig. 8. 


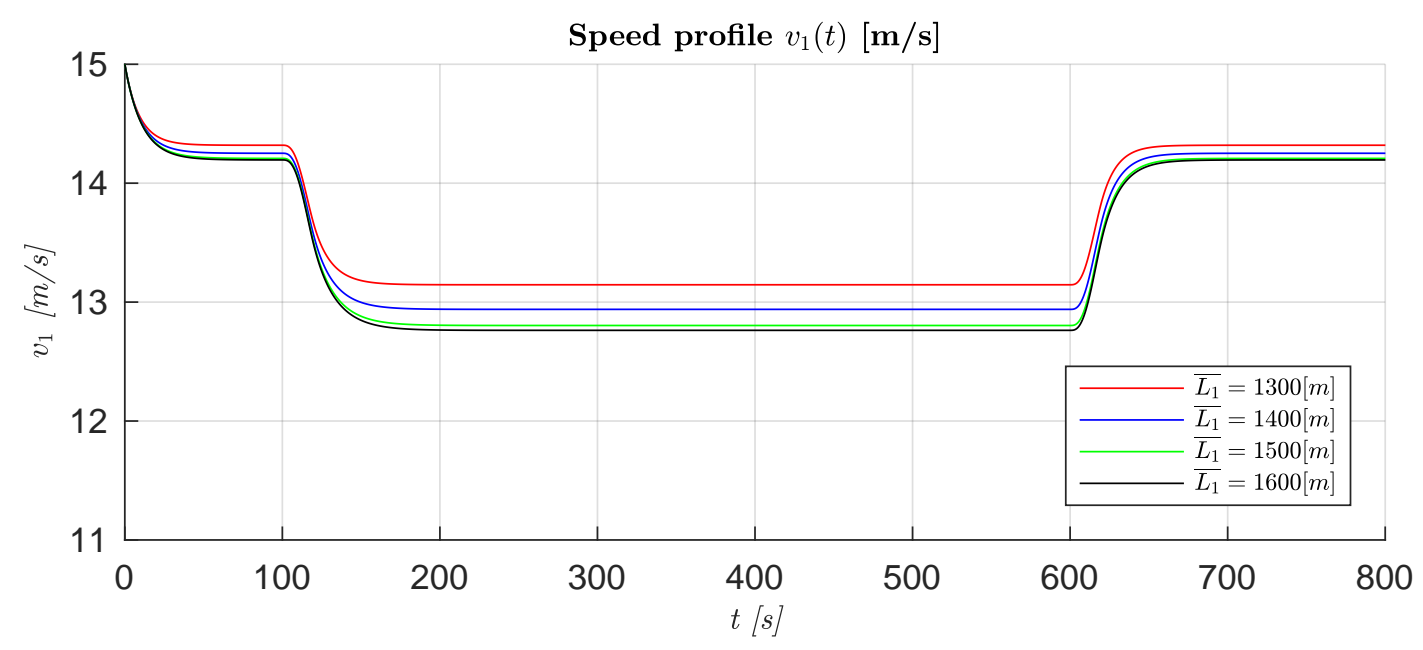

Figure 6 Evolution of the speed $v_{r}[\mathrm{~m} / \mathrm{s}]$ as a function of the simulation time $t$ [s] for Equilibrium 3 and four values of $\bar{L}_{1}=1300,1400,1500,1600$ [m].
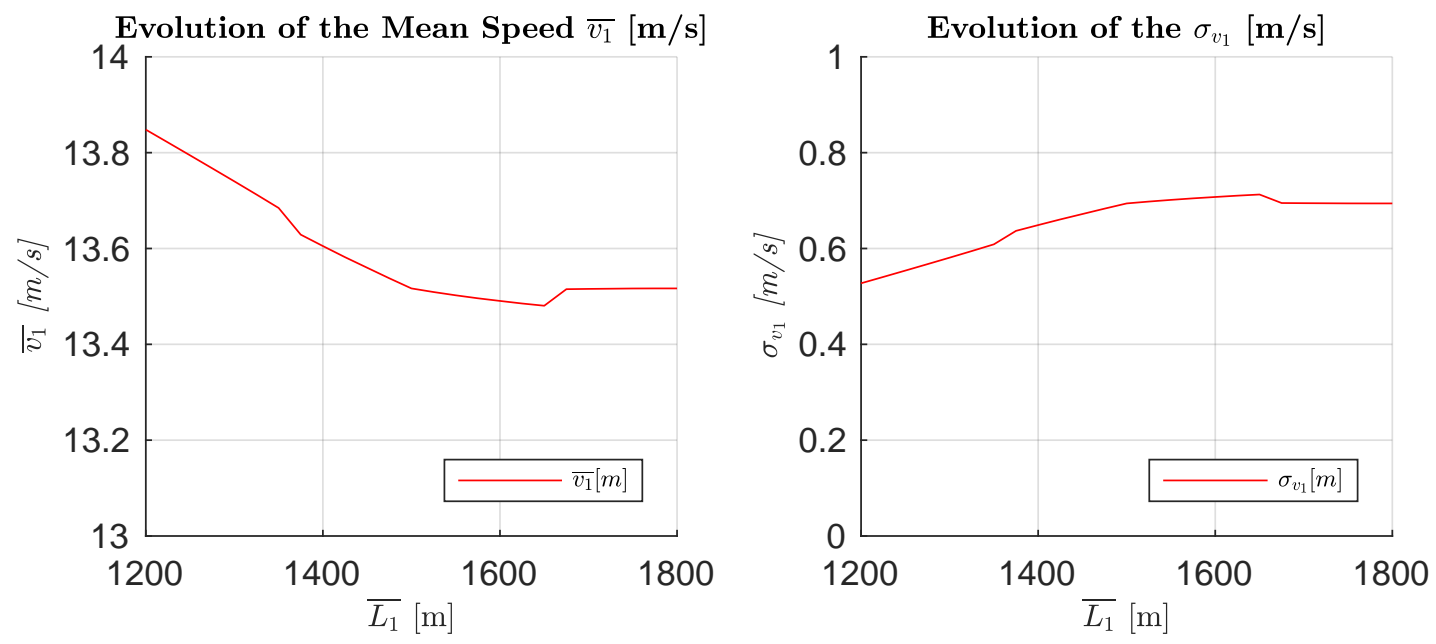

Figure 7 Evolution of the mean speed $\bar{v}_{1}[\mathrm{~m} / \mathrm{s}]$ (left panel) and of the standard deviation $\sigma_{v_{1}}$ [m] (right panel) as a function of the average trip length $\bar{L}_{1}[\mathrm{~m}]$, for Equilibrium 3 .

Thus, all vehicles choose regional path 1 , since $\alpha<0$. The average trip length for $\bar{L}_{1}=1400[\mathrm{~m}]$ is closer to $\bar{L}_{2}=1500[\mathrm{~m}]$ and the effect of $\sigma_{v_{1}}$ has an impact on the regional path flows. $U_{2}^{O D_{1}}$ is perceived as the minimal one for several Monte Carlo samples, as evidenced by the overlap between the regional path utility distributions and the $\alpha$ criterion. Hence, some vehicles choose regional path 2 (see Fig. 5). For $\bar{L}_{1} \in[1375,1475][\mathrm{m}]$, the regional paths are approximately similar because the intersection between the regional path utilities is relatively small. This is due to the fact that $\sigma_{v_{1}}$ is approximately constant as a function of $\bar{L}_{1}$ (see Fig. 7). Since all the demand of the OD pair can cross only this region, the evolutions of the traffic states are approximately similar. As $\bar{L}_{1}$ increases, vehicles switch from regional path 1 to 2 . This offsets the evolution of the speed $v_{1}$ inside the region. For $\bar{L}_{1}=\bar{L}_{2}=1500[\mathrm{~m}]$, both regional path utility distributions are equal and vehicles 


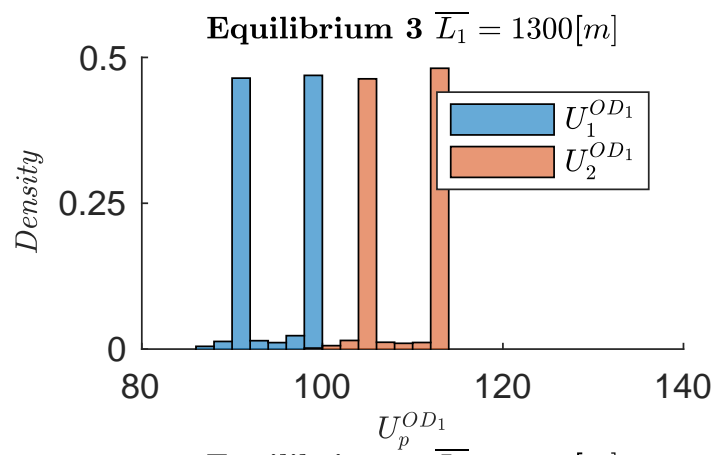

Equilibrium $3 \overline{L_{1}}=1400[\mathrm{~m}]$

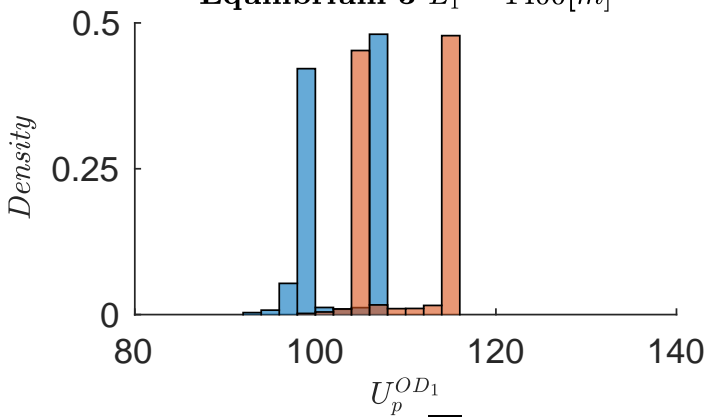

Equilibrium $3 \overline{L_{1}}=1500[\mathrm{~m}]$

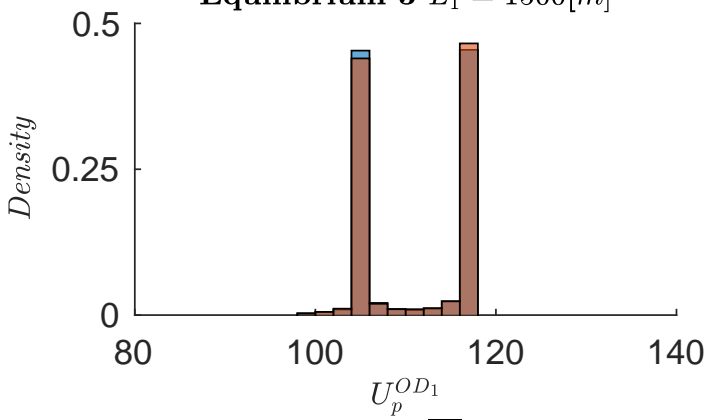

Equilibrium $3 \overline{L_{1}}=1600[\mathrm{~m}]$

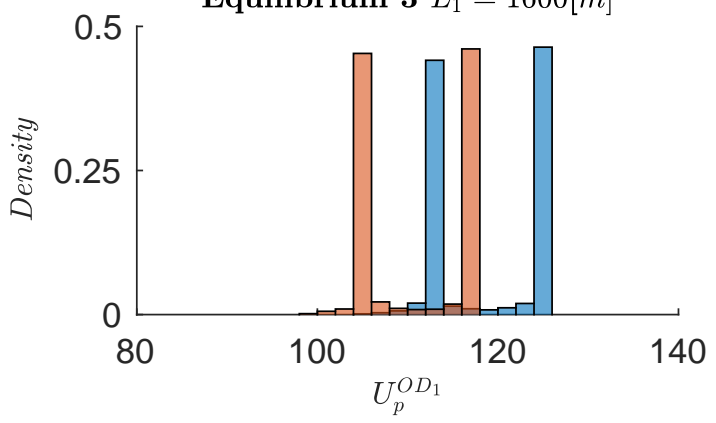

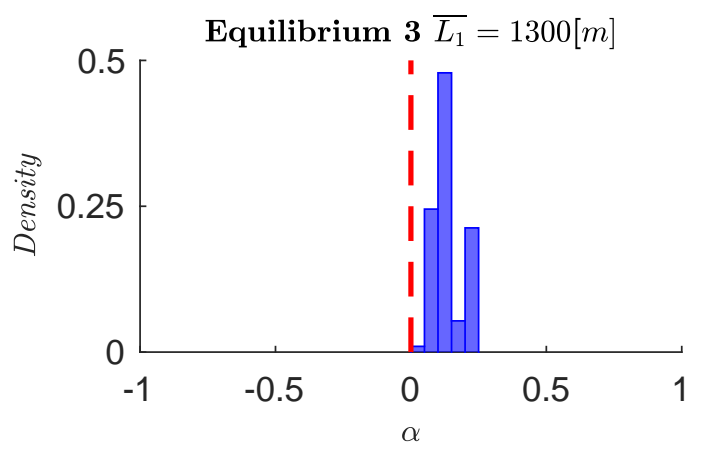

Equilibrium $3 \overline{L_{1}}=1400[\mathrm{~m}]$

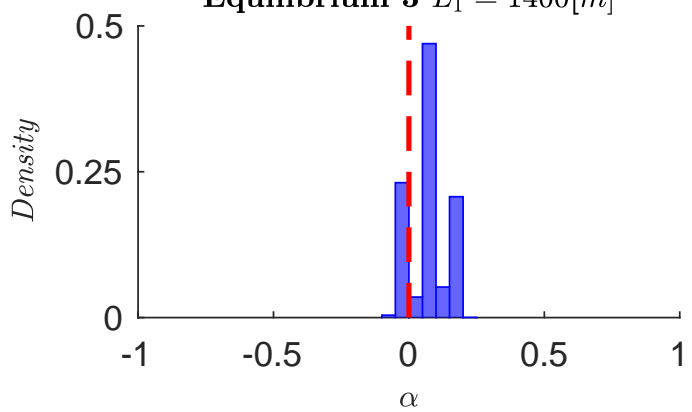

Equilibrium $3 \overline{L_{1}}=1500[\mathrm{~m}]$

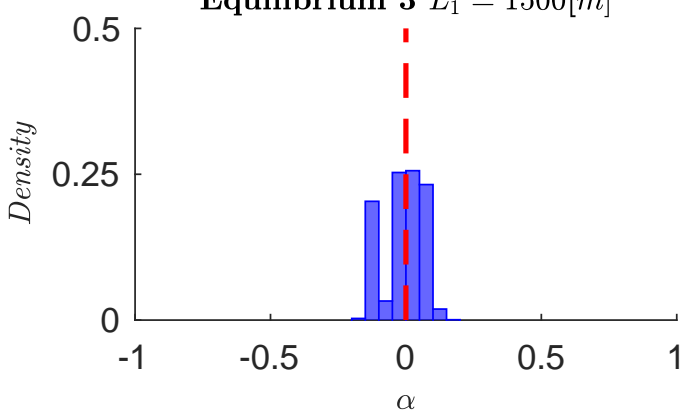

Equilibrium $3 \overline{L_{1}}=1600[\mathrm{~m}]$

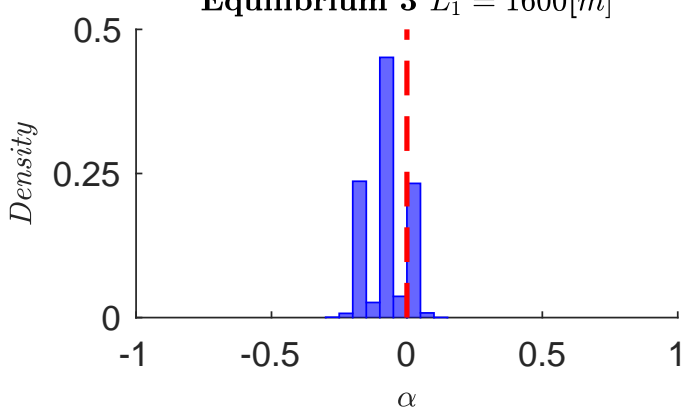

Figure 8 Left: Distributions of the regional path utilities $U_{1}^{O D_{1}}$ and $U_{2}^{O D_{1}}$. Right: $\alpha$ criterion distribution. The results are shown for Equilibrium 3 and four values of $\bar{L}_{1}[\mathrm{~m}]$. The vertical red dashed line represents the value at $\alpha=0$. 

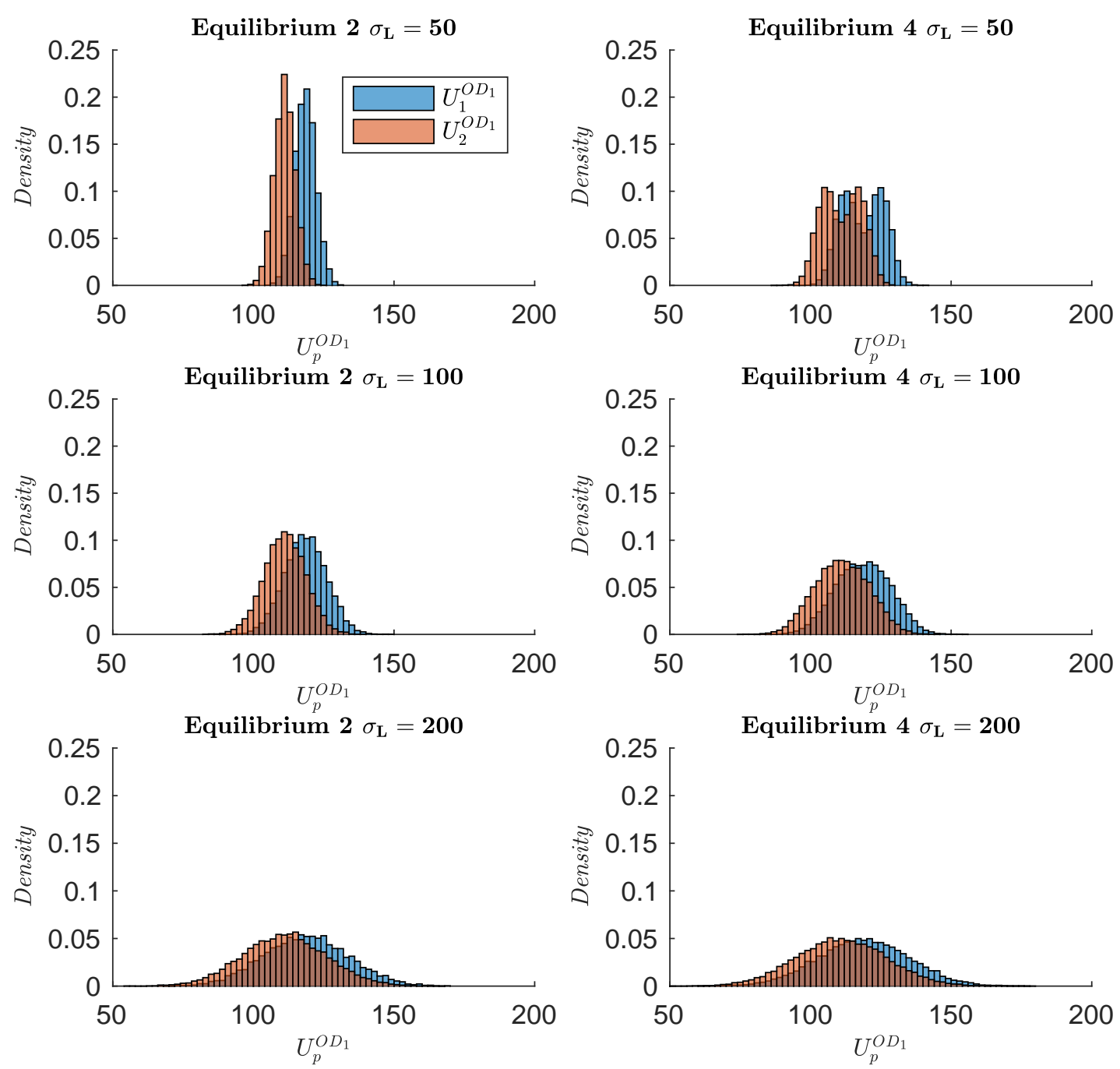

Figure 9 Regional path utility distributions $U_{1}^{O D_{1}}$ and $U_{2}^{O D_{1}}$, for Equilibrium 2 (left) and 4 (right) and $\bar{L}_{1}=1600$ [m]. The results are shown for the three values of $\sigma_{L}$.

choose both regional paths equally as shown in Fig. 5. For $\bar{L}_{1}=1600[\mathrm{~m}]$, the distribution of $U_{1}^{O D_{1}}$ is shifted towards larger values compared to $U_{2}^{O D_{1}}$ due to the increase of $\bar{L}_{1}$. As $\bar{L}_{1}$ increases, vehicles that choose regional path 1 need more time to complete their trips inside the region, reducing the speed $v_{1}$. This shifts the distribution of $U_{1}^{O D_{1}}$ towards higher values compared to the distribution of $U_{2}^{O D_{1}}$. Thus, around $80 \%$ of the vehicles choose regional path 2 . For larger values of $\bar{L}_{1} \geq 1700$ $[\mathrm{m}]$ all vehicles choose regional path 2 since $U_{1}^{O D_{1}}>U_{2}^{O D_{1}}$. The evolution of the regional path flows as $\bar{L}_{1}$ increases for Equilibrium 3 is completely different from that of Equilibrium 4 for the three $\sigma_{L}$ values considered (see Fig. 5). This enhances the importance of taking into account the trip length distributions, when calculating the regional path flows distributions. 

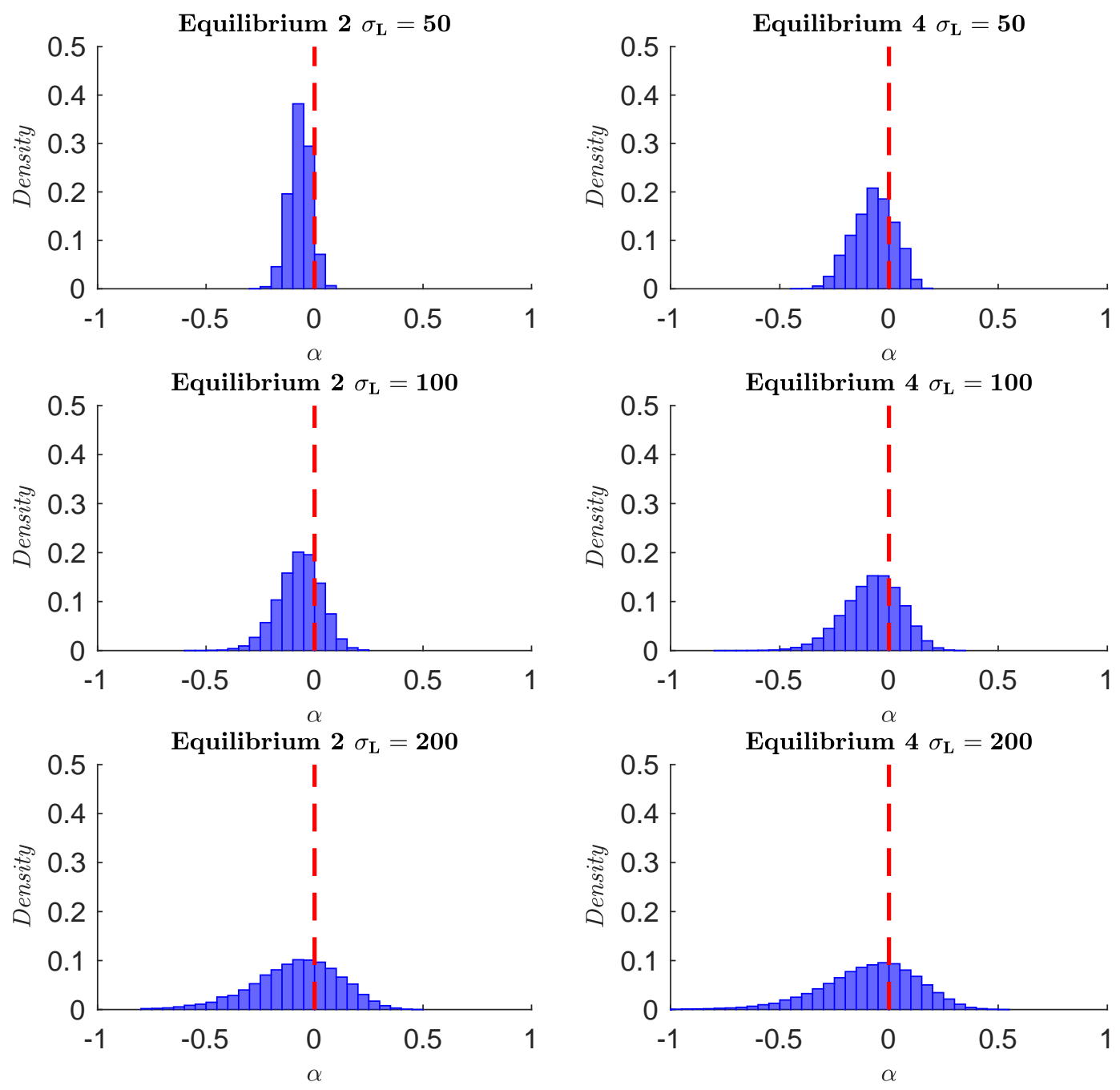

Figure $10 \alpha$ distributions for Equilibrium 2 (left) and 4 (right), $\bar{L}_{1}=1600$ [m] and three values of $\sigma_{L}=50,100$ and $200[\mathbf{m}]$. The vertical red dashed line represents the value at $\alpha=0$.

Equilibrium 2 considers that only the trip lengths $L_{r p}$ are distributed. Equilibrium 2 and 4 give similar regional path flows for larger values of $\sigma_{L}=200$. This is because the influence of $\sigma_{v_{1}}$ becomes less significant for large $\sigma_{L}$ values. For the three $\sigma_{L}$ values and for $\bar{L}_{1}=\bar{L}_{2}=1500[\mathrm{~m}]$, the regional path utilities of Equilibrium 2 and 4 are similar. Consequently, vehicles choose both regional paths equally. To investigate the similarities and differences between Equilibrium 2 and 4 in more detail, we fix $\bar{L}_{1}=1600[\mathrm{~m}]$ and analyze the regional path utility distributions (Fig. 9) for the three values of $\sigma_{L}$. We also consider the respective distributions of $\alpha$ (Fig. 10). The similarities between both network equilibria depend on the standard deviation $\sigma_{L}$ and on the position of the two peaks of the regional path utilities for Equilibrium 3 compared to those of Equilibrium 2 (see e.g. Fig. 9). This is 
because the reference Equilibrium 4 is a convolution of Equilibrium 2 and 3 regional path utilities (see Eq. 9). For $\sigma_{L}=50[\mathrm{~m}]$, the convolution between the regional path utilities of Equilibria 2 and 3, leads to a bi-variate shape for the regional path utilities of Equilibrium 4 (Fig. 9). The latter occurs since the $\sigma_{L}$ value is not sufficiently large to offset the distance between the two peaks of the regional path utilities of Equilibrium 3, i.e. the effect due to $\sigma_{v_{1}}$. For larger $\sigma_{L}=200[\mathrm{~m}]$ values, the $\sigma_{L}$ offsets the influence of $\sigma_{v_{1}}$ and the regional path flows between Equilibrium 2 and 4 are similar. These two effects offset each other and lead to similar regional path flows between Equilibria 2 and 4 , as also shown by the $\alpha$ criterion. For larger $\sigma_{L}=100,200[\mathrm{~m}]$ values, these two peaks vanish from the regional path utilities as observed in Fig. 9. Nevertheless, after convolution, they still control where the largest fraction of the regional path utilities for Equilibrium 4 will be located. For the case shown in Fig. 9, the overlap between $U_{1}^{O D_{1}}$ and $U_{2}^{O D_{1}}$ is similar for both Equilibrium 2 and the reference Equilibrium 4. Therefore, as also evidenced by the $\alpha$ criterion, the regional path flows are similar between both regional paths.

Equilibrium 2 gives the regional path flow distributions closest to those of Equilibrium 4, but the differences become more significant for lower values of $\sigma_{L}$, showing the importance of also considering that $v_{r}$ is distributed. Despite the regional path flows between Equilibrium 2 and 4 are similar for this test case and larger values of $\sigma_{L}=200[\mathrm{~m}]$, this might not be true when there are also interactions between different regional OD pairs. This is investigated in the next section. In brief, we cannot neglect the variability of trip lengths and traffic states inside the region. The variability of $v_{r}$ is particularly relevant for heterogeneous networks consisting of a mix of different road categories (e.g. local roads and urban motorways). If we do so, we may obtain regional path flows that are very different from those given by the reference Equilibrium 4.

\subsection{Analysis of the regional path flows at equilibrium: the 2-region test case}

In this section, we investigate how different the regional path flows calculated for the reference Equilibrium 4 are compared to the other three network equilibria for a more complex two-region test case. The trip lengths for the four regional paths are also sampled according to a normal distribution with: fixed mean lengths $\bar{L}_{1}=1400, \bar{L}_{2}=1500$ and $\bar{L}_{4}=1700[\mathrm{~m}]$ for regional paths 1 , 2 and 4, respectively; and an increasing mean trip length $\bar{L}_{3}$ between 1200 and 2200 [m] varying stepwise by $25[\mathrm{~m}]$. We also consider three values of the standard deviation $\sigma_{L}=50,100,200[\mathrm{~m}]$.

We focus our analysis on the regional $O D_{2}$ pair since it is the most interesting one. In this test setting, all the demand of the regional $O D_{1}$ passes through region $R_{1}$. This influences the regional path flows of $O D_{2}$, since regional path 3 passes through region $R_{1}$.

In Fig. 11, we show the path flows as a function of $\bar{L}_{3}$, for regional paths 3 and 4 and the four network equilibria. First, it can be observed that the flows of regional paths 3 and 4 are not equally 

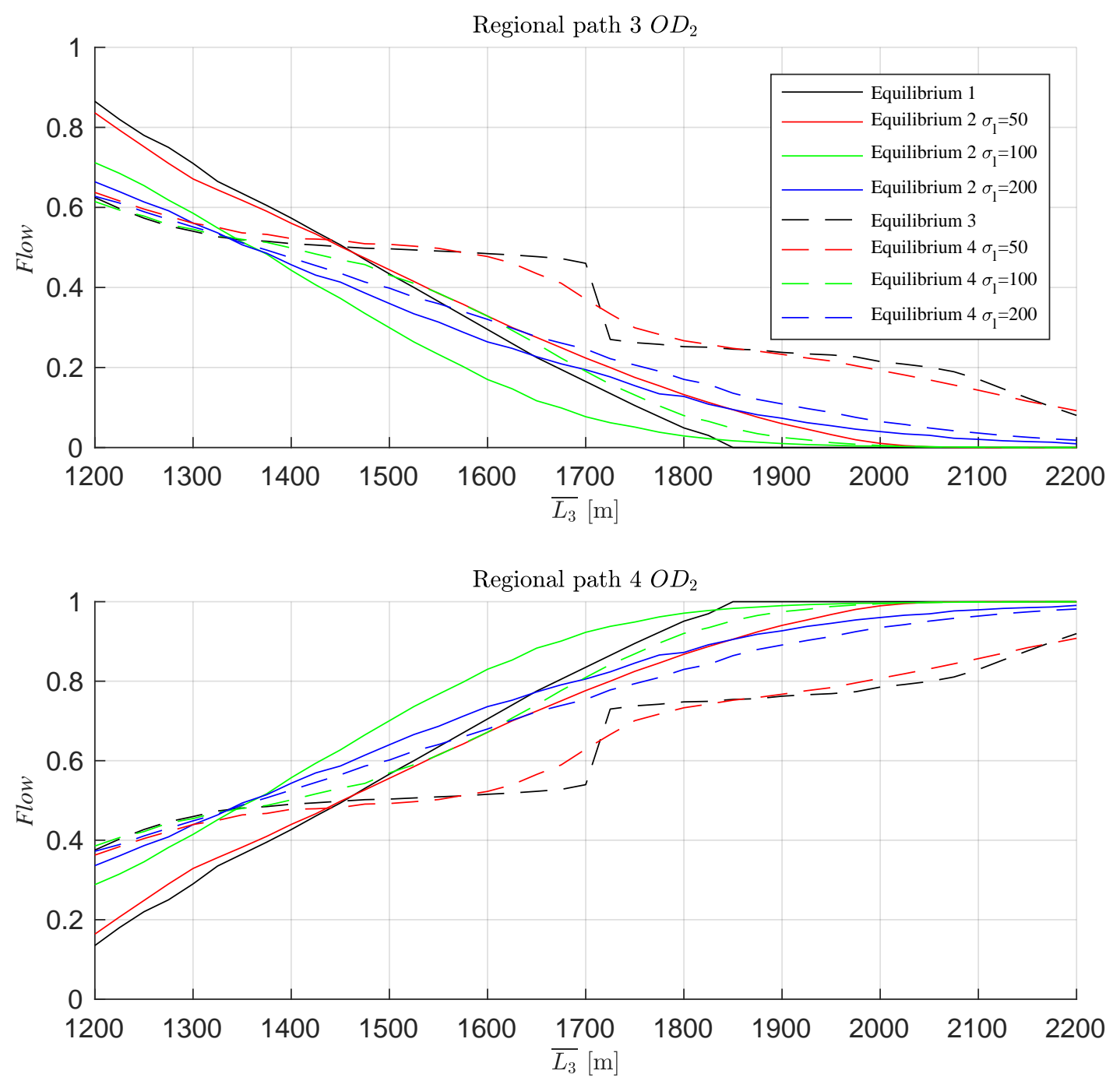

Figure 11 Path flows as function of $\bar{L}_{3}$, for regional paths 3 and 4 that connect the $O D_{2}$ pair. For Equilibria 2 and 4 , three values of $\sigma_{L}$ are considered.

distributed when $\bar{L}_{3}=\bar{L}_{4}=1700[\mathrm{~m}]$, due to the interaction with the $O D_{1}$. The regional path flows are equally distributed for different values of $\bar{L}_{3}$, depending on the network equilibrium. Second, the regional path flows for Equilibria 1 and 3 are those that differ most in comparison to the reference Equilibrium 4. We start by analyzing Equilibrium 1 in more detail. Vehicles switch from regional path 3 to 4 as $\bar{L}_{3}$ increases, such that the condition $U_{3}^{O D_{2}}=U_{4}^{O D_{2}} \Rightarrow \frac{\bar{L}_{3}}{\bar{L}_{4}}=\frac{\bar{v}_{1}}{\bar{v}_{2}}$ is satisfied. To analyze the differences and similarities between Equilibria 2 and 3 compared to the reference Equilibrium 4, we analyze the regional path flows for a fixed value of $\bar{L}_{3}=1850$ [m]. To do this, we consider the distributions of the regional path utilities $U_{3}^{O D_{2}}$ and $U_{4}^{O D_{2}}$ for $\bar{L}_{3}=1850[\mathrm{~m}]$. The results are shown in Fig. 12. 

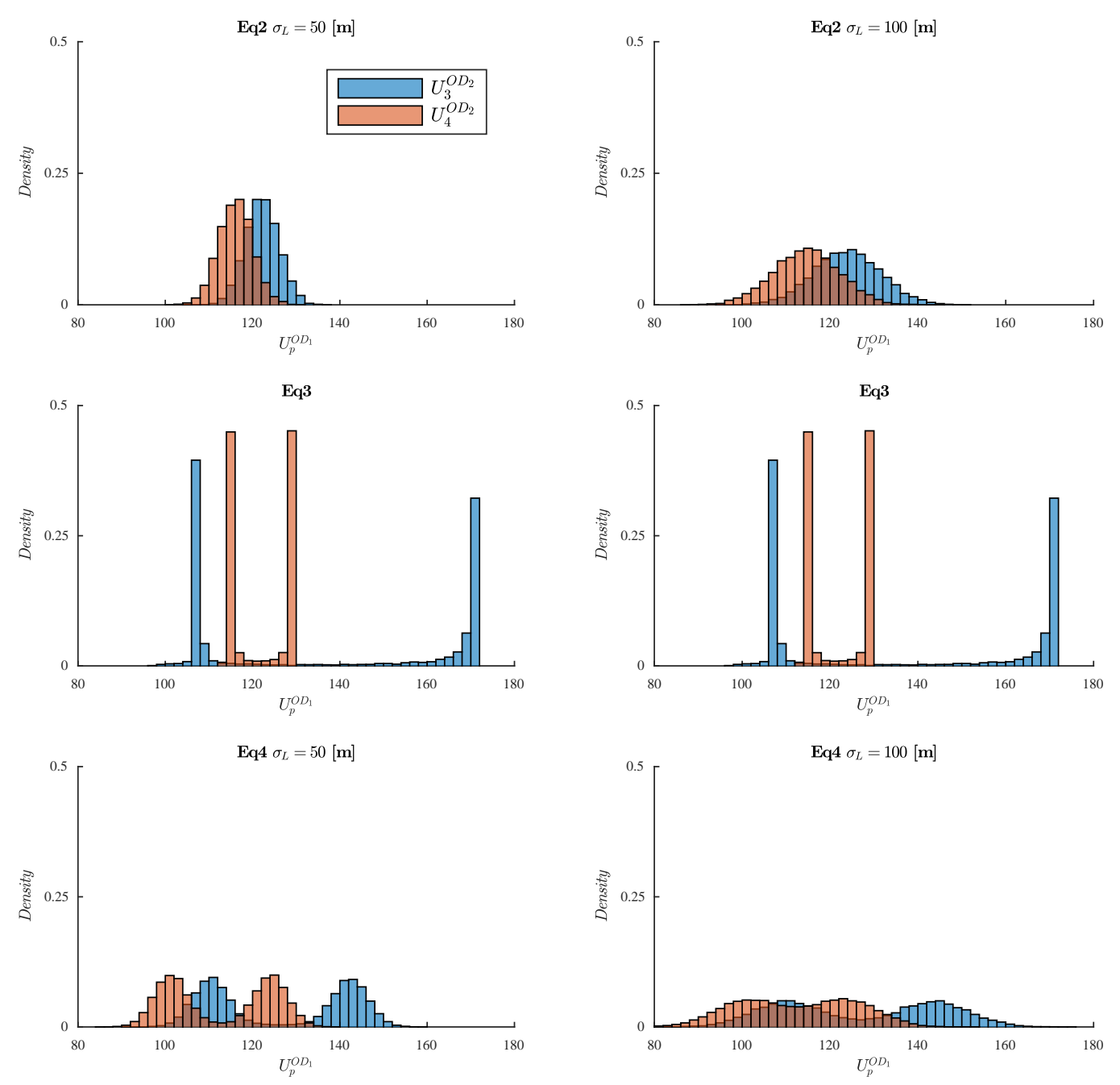

Figure 12 Distributions of the regional path utilities $U_{3}^{O D_{2}}$ and $U_{4}^{O D_{2}}$ for Equilibrium 2 to 4 . The results are shown for $\bar{L}_{3}=1850$ [m]. For Equilibrium 2 and 4, two values of $\sigma_{L}=50,100$ [m] are considered.

As with the analysis of the previous section test case, the shape and overlap between $U_{3}^{O D_{2}}$ and $U_{4}^{O D_{2}}$ for the reference Equilibrium 4 depend on the position of the distribution peaks for Equilibrium 3 compared to the positions of the distributions for Equilibrium 2, and on the standard deviation $\sigma_{L}$. We first consider the case of $\sigma_{L}=50[\mathrm{~m}]$, in Fig. 12. The convolution between the regional path utility distributions of Equilibria 2 and 3 leads to a bivariate distribution for Equilibrium 4. The closeness between the two peaks of different regional path utility distributions for Equilibrium 3, induces a large overlap between $U_{3}^{O D_{2}}$ and $U_{4}^{O D_{2}}$ for Equilibrium 4. Since this overlap is larger for the reference Equilibrium 4 compared to Equilibrium 2, the regional path utility $U_{3}^{O D_{2}}$ might be perceived as the minimal one for a larger number of Monte Carlo trials. 
Consequently, for $\bar{L}_{3}=1850[\mathrm{~m}]$, the path flow of regional path 3 is slightly larger for the reference Equilibrium 4 than for Equilibrium 2. But the effects of the convolution between the regional paths utility distributions of Equilibria 2 and 3 are offset for larger values of $\sigma_{L}$. For Equilibrium 3 , the regional path utility distributions are similar for $\sigma_{L}=50$ and $\sigma_{L}=100$, since they do not depend on $\sigma_{L}$. But for larger values of $\sigma_{L}$ and for Equilibrium 2, the intersection between $U_{3}^{O D_{2}}$ and $U_{4}^{O D_{2}}$ increases. This increases the chance that $U_{3}^{O D_{2}}$ is perceived as the minimal utility for a larger number of Monte Carlo trials. In this case, it leads to an approach between the regional path flows calculated for Equilibria 2 and 4. In brief, Equilibrium 2 is the one which gives the regional path flows closest to the reference Equilibrium 4. However, it is difficult to predict in which circumstances this will occur. This backs the conclusion of the previous section, where we should take into account the effects of the trip length variability as well as the time-evolution of regional traffic states.

\subsection{The effect of the correlation between regional paths on the network equilibrium}

In this section, we show that the framework proposed takes into account the correlation between regional paths, thanks to the Monte Carlo trials performed at the region level. To this end, we compare the regional path flows calculated for Equilibria 2 and 4 with the Multinomial Logit model. The MNL assumes that the distribution of travel times over paths are independent and identically Gumbel distributed, with a scale parameter $\theta$. Following Chen et al. (2012), the scale parameter is defined at the origin-destination od level, in city networks. In this paper, use the regional OD level as baseline, i.e. $\theta^{O D}$. The MNL model has the advantage of having a closed form for the calculation of the regional paths' choice probability. However, the MNL is not able to handle the correlation between regional paths due to the $\theta^{O D}$ assumption.

We consider two test scenarios. The first test scenario corresponds to the 1-region network and demand scenario shown in Fig. 3. The trip lengths are sampled according to a normal distribution with a fixed mean $\bar{L}_{2}=1500[\mathrm{~m}]$ for regional path 2 ; and a mean value varying between 1300 to $1700[\mathrm{~m}]$, increasing the step size by 25 [m] for regional path 1 . The standard deviations for both regional paths are fixed at $\sigma_{L}=50,100,200[\mathrm{~m}]$. The second test scenario corresponds to the 2-region network and demand scenario shown in Fig. 4 (a). The trip lengths are sampled according to a normal distribution with: fixed mean lengths $\bar{L}_{1}=1400, \bar{L}_{2}=1500$ and $\bar{L}_{4}=1700[\mathrm{~m}]$ for regional paths 1, 2 and 4, respectively; and an increasing mean trip length $\bar{L}_{3}$ between 1200 and $2200[\mathrm{~m}]$ varying stepwise by $25[\mathrm{~m}]$. The standard deviations for the four regional paths are fixed at $\sigma_{L}=50,100,200[\mathrm{~m}]$. 

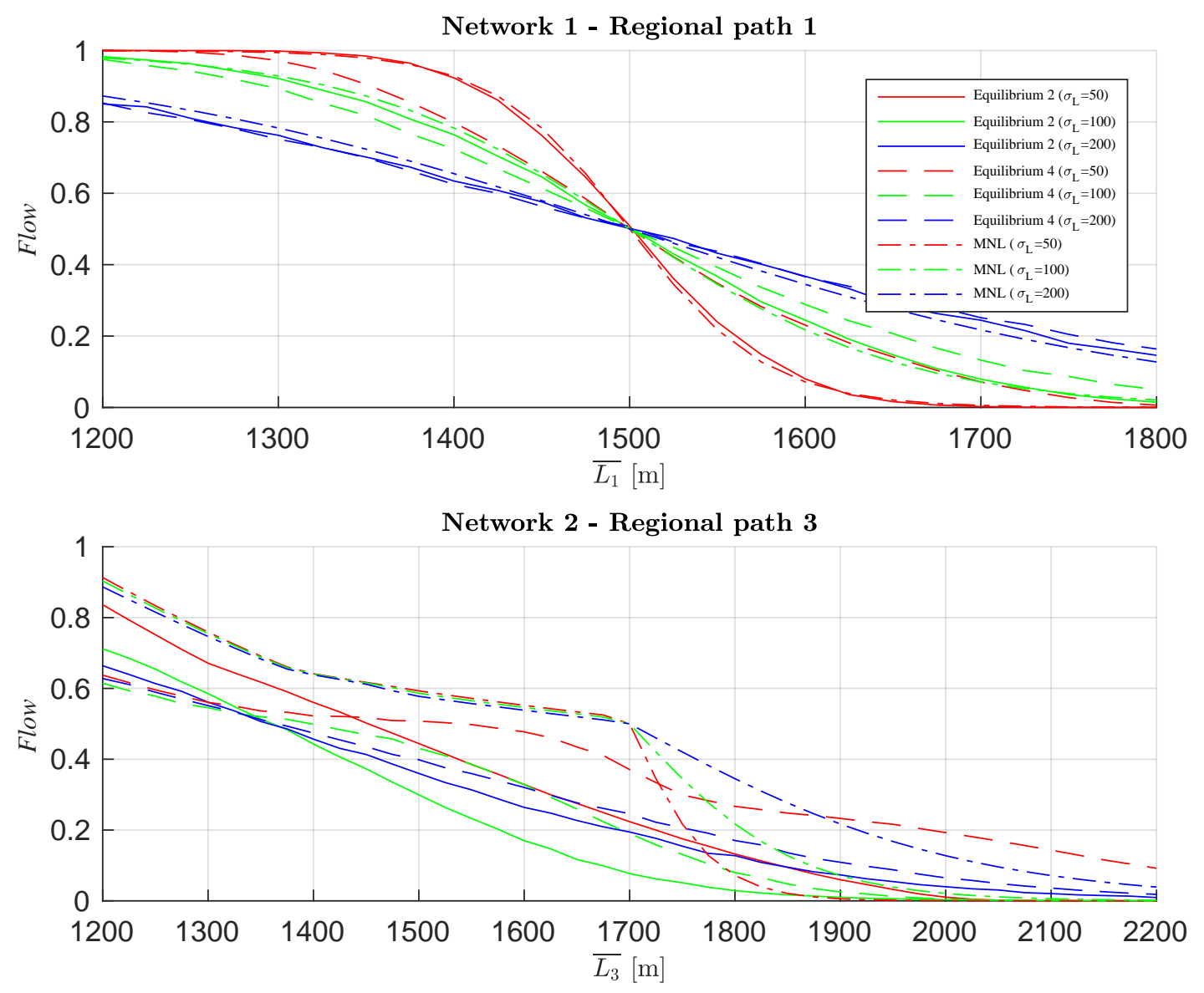

Figure 13 Top: Regional path flows at equilibrium as a function of $\bar{L}_{1}$, for the first test scenario. Bottom: Regional path flows at equilibrium as a function of $\bar{L}_{3}$, for the second test scenario.

The scale parameter of the MNL, $\theta^{O D}$, is calibrated according to the previous values of $\sigma_{L}[\mathrm{~m}]$, as:

$$
\theta^{O D}=\sqrt{\frac{\pi^{2} \bar{v}_{r}^{2}}{6 \sigma_{L}^{2}}}
$$

In Fig. 13, we show the regional path flows for both test scenarios, for Equilibria 2 and 4, and for the MNL. For the first test scenario, we observe that the regional path flows for Equilibrium 2 are similar to those of the MNL. This occurs because we consider an independent sampling of trip lengths, with a similar $\sigma_{L}$ value for both regional paths. Moreover, the utility function defined in Eq. 7 to calculate Equilibrium 2 does not consider that the regional mean speed set $v_{r}\left(n_{r}\right)$ is distributed and that the average mean speed $\bar{v}_{r}$ is the same for both regional paths. On the other hand, the calculation of Equilibrium 4 considers that both the mean speed and trip lengths are distributed, capturing the correlation between the two regional paths. This is demonstrated by the 
different regional path flows for Equilibrium 4 and the MNL shown in Fig. 13. For large $\sigma_{L}$ values, the influence of $\sigma_{v}$ is offset. Therefore, the regional path flows are similar for Equilibrium 2 and 4 and the MNL. Whilst, for low $\sigma_{L}$ values, $\sigma_{v}$ plays an important role and the regional path flows for Equilibrium 2 and the MNL are different than those of the reference Equilibrium 4. For the second test scenario, it can be seen that Equilibrium 2 gives regional path flows different from those of the MNL. This occurs because in this test scenario, we have a correlation between regional paths connecting different regional OD pairs. The latter influences the calculation of the mean speed $\bar{v}_{r}$ for this region and directly influences the utility function defined in Eq. 7. On the other hand, the scaling of the $\theta^{O D}$ of the MNL is not sensitive to the mean speed $\bar{v}_{r}, \forall r=1,2$.

In brief, we show the importance of considering the effect of the traffic states in the calculation of the network equilibrium, to capture the correlation between regional paths. Moreover, we show that the reference Equilibrium 4 represents an extension of the dynamic traffic assignment framework for regional networks proposed by Yildirimoglu and Geroliminis (2014). First, our framework accounts for distributions of trip lengths that are explicitly calculated, while Yildirimoglu and Geroliminis (2014) calculated them in an iterative process, as discussed in the introduction. Second, we do not assume any prior statistical distribution for the regional path travel times. Yildirimoglu and Geroliminis (2014) assumed that these terms are identically and independently distributed Gumbel variables and made use of the MNL formulation. Third, our framework captures the correlation between regional paths, while the MNL formulation does not.

\section{Application to a real test case: the $6^{\text {th }}$ district of the Lyon network}

In this section, we demonstrate the importance of considering $\sigma_{L}$ and $\sigma_{v}$ in the definition of the utility function for a real setting related to a real city network. We start by introducing the city network and test scenarios (Sect. 4.1). We then analyze the influence of $\sigma_{L}$ and $\sigma_{v}$ on the network equilibrium considering two different scenarios. In the first one, we analyze the individual influence of the previous two factors on the traffic dynamics in the regions at equilibrium (Sect. 4.2). In the second one, we analyze their combined influence on the traffic dynamics at equilibrium (Sect. 4.3). In particular, we discuss the role of $\sigma_{v}$ in the calculation of the network equilibrium.

\subsection{Definition of the test scenarios}

The traffic states are simulated through an accumulation-based MFD model (Daganzo 2007, Geroliminis and Daganzo 2008) for the $6^{\text {th }}$ district of the Lyon network (Fig. 14 (a)). This network has 757 links and 431 nodes and is divided into 8 regions. The MFD functions (Fig. 14 (b)) have been fitted considering microscopic simulations from Symuvia (Leclercq 2007) on the same network, where the demand scenario mimics a morning peak. We further assume a bi-parabolic shape to fit the simulated data. 

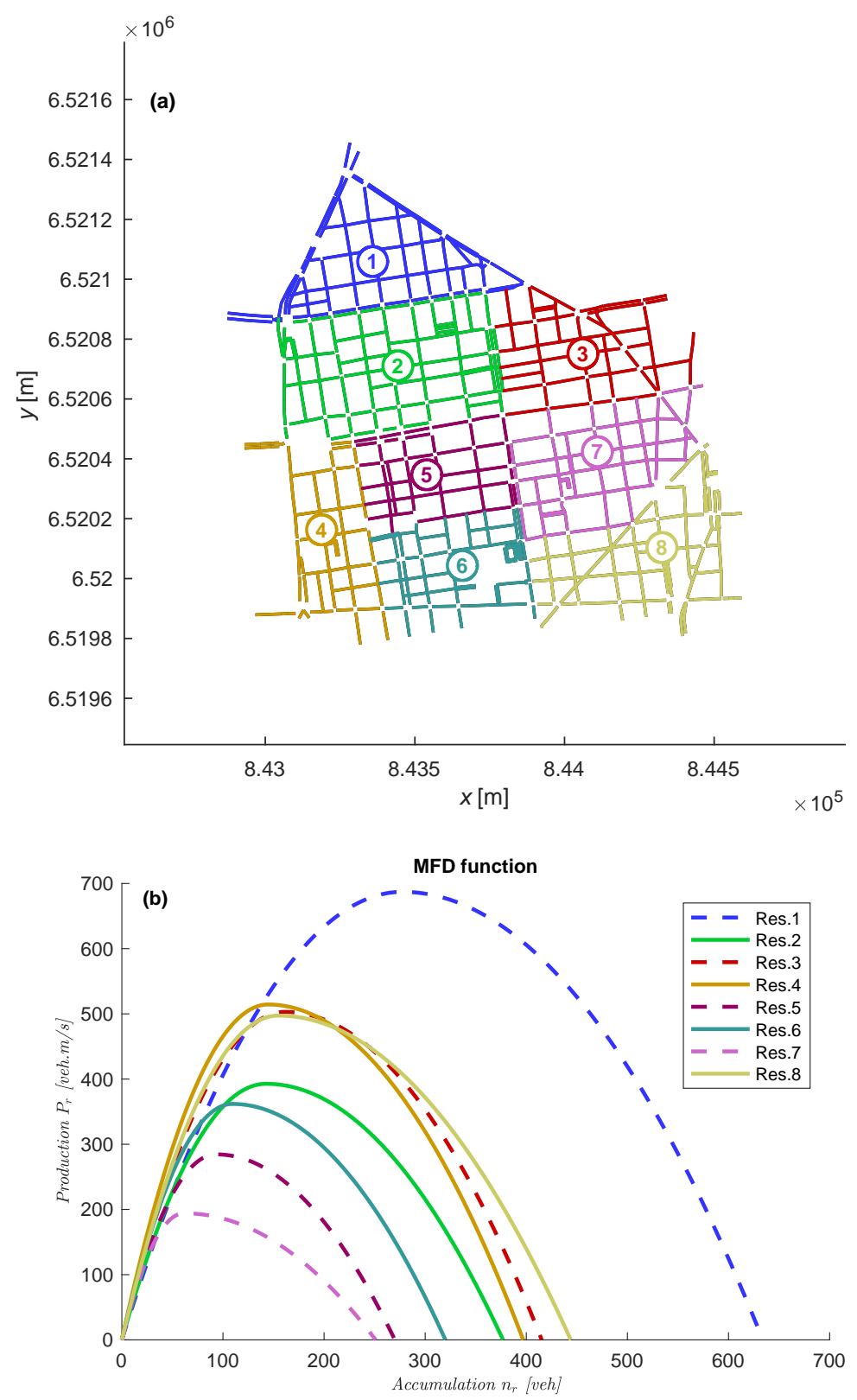

Figure 14 (a) Lyon $6^{\text {th }}$ district network divided into 8 regions. (b) MFD function of each region.

The regional choice set $\Omega^{O D}$ (Batista et al. 2018) and the distributions of trip lengths $L_{r p}$ (Batista, Leclercq, and Geroliminis 2019) that characterize the regional paths are explicitly calculated following the procedure previously discussed in Sect. 2.2. We randomly sample $N_{\text {od }}=10000$ od pairs on the $6^{\text {th }}$ district Lyon network and calculate the shortest-path in distance for each one. Each trip represents an indivual travel in the city network. This step allows to define the set of virtual trips $\Gamma$. We then consider the definition of the city network partitioning and verify the sequence of regions that each virtual trip crosses, i.e. the path they define on the regional network or also called as regional path. The virtual trips are then grouped by the regional path they define. 

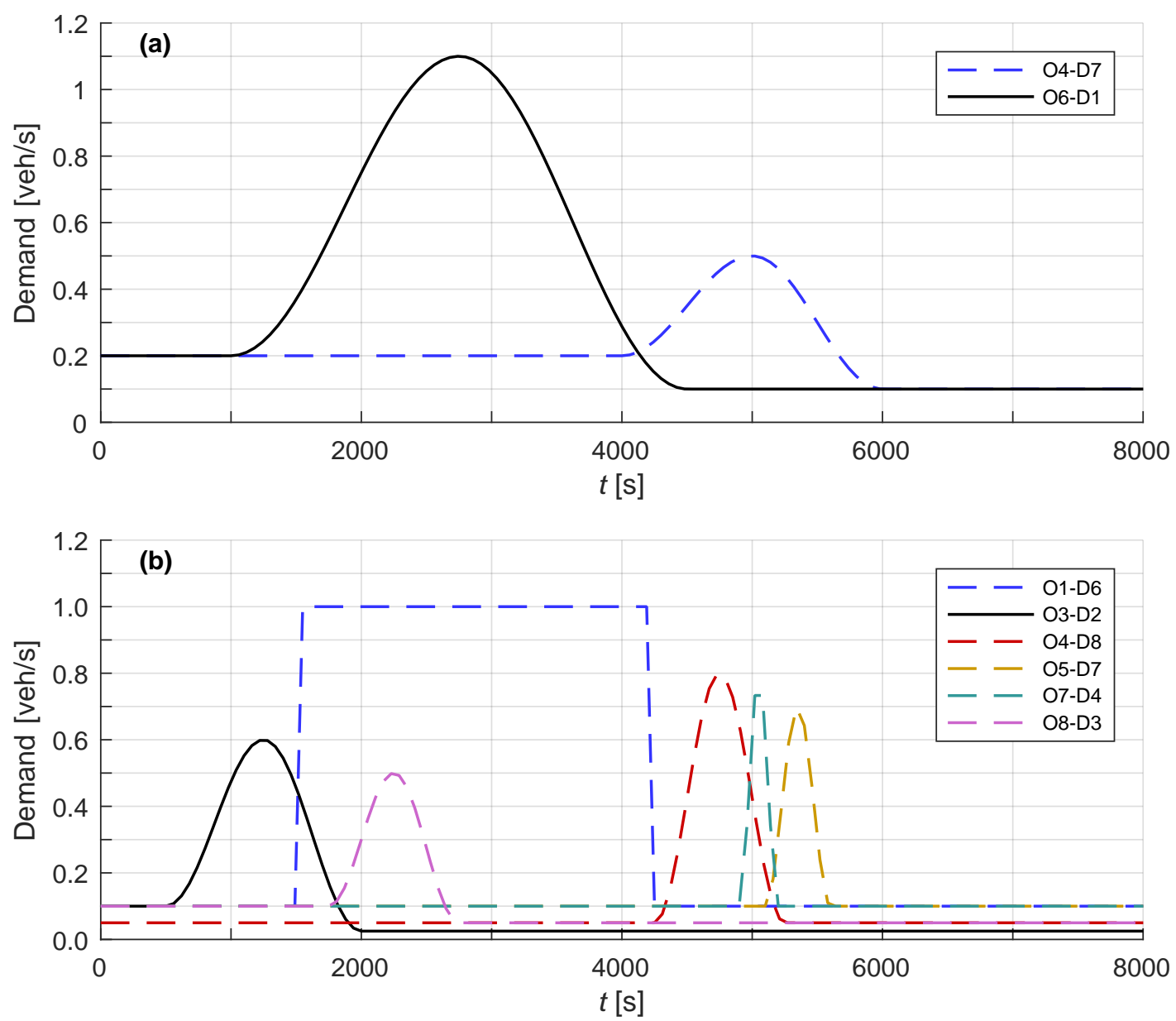

Figure 15 (a) Demand scenario 1 with 2 OD pairs. (b) Demand scenario 2 with 6 OD pairs.

We are left in total with 205 regional paths. They are ranked with different levels of significance, i.e. different regional paths are defined by a different number of virtual trips. We define the most significant regional paths for one OD pair as those that are defined by the largest number of virtual trips. In this paper, we assumes a maximum of the two most significant regional paths of each OD pair to gather the regional choice set $\Omega^{O D}$. The distributions of trip lengths are calculated based on the travel distances inside each region, of all virtual trips associated to the same regional path (Batista, Leclercq, and Geroliminis 2019). The set of trip lengths of regional path $p$ inside region $r$ is mathematically described as (Batista, Leclercq, and Geroliminis 2019):

$$
L_{r p}=\left\{\delta_{r k}^{p} l_{r k}\right\}, \forall k \in \Gamma
$$

where $l_{r k}$ is the length of trip $k$ inside region $r$; and $\delta_{r k}^{p}$ is a binary variable that equals 1 if trip $k$ crosses region $r$ and is associated to regional path $p$, or 0 otherwise.

As previously stated, in this section we investigate the influence of $\sigma_{L}$ and $\sigma_{v}$ on the traffic dynamics in the regions at equilibrium, in two distinct scenarios. In the first scenario (scenario 1), 
we investigate the individual influence of the previous two factors on the traffic dynamics in the regions at equilibrium conditions. We consider two OD pairs: 4-7; and 6-1. We have a total of 4 regional paths. Table 2 lists the regional paths and their average trip lengths in each region for this scenario 1. In the second scenario (scenario 2), we investigate the influence of the combined contributions of $\sigma_{L}$ and $\sigma_{v}$ on the traffic dynamics in the regions. We consider 6 regional OD pairs: 1-6; 3-2; 4-8; 5-7; 7-4; and 8-3. There are 17 regional paths in total. Fig. 15 depicts the demand curves for these two scenarios.

Table 2 Average regional trip lengths $(m)$ for the regional paths for the demand scenario 1.

\begin{tabular}{|c|ccccccccc|}
\hline \multirow{2}{*}{ Regional path } & \multicolumn{7}{|c|}{ Region } \\
\cline { 2 - 8 } & 1 & 2 & 3 & 4 & 5 & 6 & 7 & 8 \\
\hline $4-5-7$ & $\sim$ & $\sim$ & $\sim$ & 246 & 464 & $\sim$ & 387 & $\sim$ \\
$4-6-8-7$ & $\sim$ & $\sim$ & $\sim$ & 234 & $\sim$ & 414 & 130 & 464 \\
$6-5-2-1$ & 242 & 427 & $\sim$ & $\sim$ & 279 & 174 & $\sim$ & $\sim$ \\
$6-4-2-1$ & 196 & 355 & $\sim$ & 318 & $\sim$ & 265 & $\sim$ & $\sim$ \\
\hline
\end{tabular}

The total simulation period is set to $T=8000$ seconds and is divided into 32 assignment periods of 250 seconds each. The network equilibrium is calculated for each period. For the convergence criteria, we consider a maximum number of violations of $\Phi=0$ and/or a Gap tolerance $t o l \leq 0.01$ and a maximum of descent step iterations of $N_{\max }=100$. For the Monte Carlo simulations, we consider $N_{L_{r p}}^{M C}=N_{v_{r}}^{M C}=10000$ samples.

\subsection{Analysis of the individual influence of $\sigma_{L}$ and $\sigma_{v}$ on the network equilibrium}

In this section, we investigate the individual influence of $\sigma_{L}$ and $\sigma_{v}$ on the calculation of the network equilibrium. Fig. 16 shows the traffic states modeled for scenario 1 and for the eight regions of the $6^{\text {th }}$ district of the Lyon network. Fig. 17 depicts the regional path flows at equilibrium. The results are shown for the four network equilibria. The analysis of $\sigma_{L}$ and $\sigma_{v}$ in the calculation of the network equilibrium on a real network is more challenging than in the theoretical networks discussed in the previous Sect. 3. This is because of the larger heterogeneity of average trip lengths and $\sigma_{L}$ assigned to the different regional paths (Batista, Leclercq, and Geroliminis 2019). As discussed by Batista, Leclercq, and Geroliminis (2019), this directly influences the traffic dynamics since a larger travel distance for one path inside a given region means a potential bottleneck. As an example, let's consider two regional paths that are crossing the same region. One of the regional paths has an average travel distance of 500 meters inside this region, while the other one has 1000 meters. The vehicles traveling on the longer regional path need more time to complete their trips inside this region because of the speed-MFD assumption, i.e. all vehicles travel inside the same region at the same average speed for a given time instant. This increases the vehicles accumulation for a 

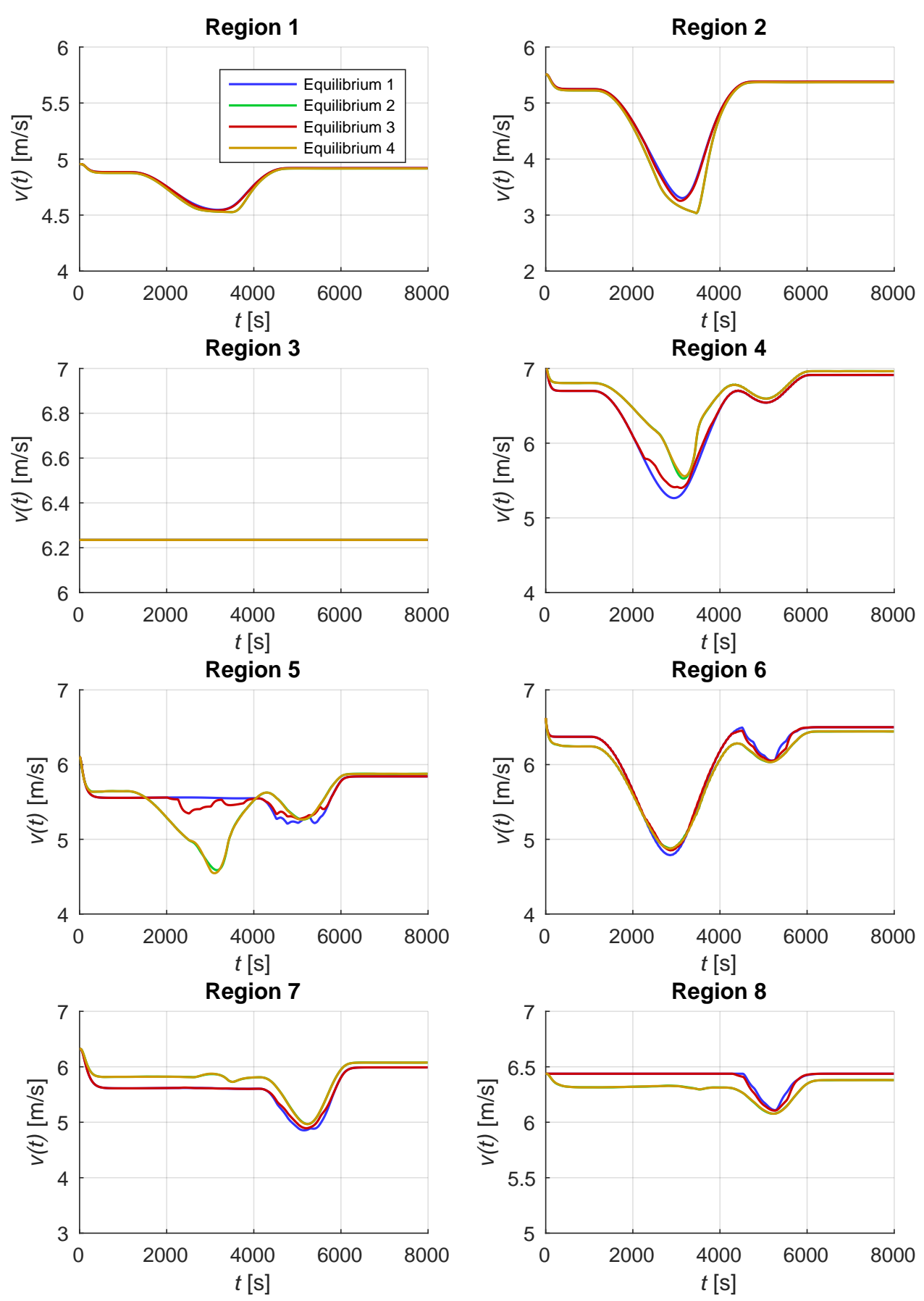

Figure 16 Evolution of the mean speed $v(t)$ as a function of the simulation time $t$ for the eight regions and the four network equilibria. The results depicted are for the demand scenario 1 . 

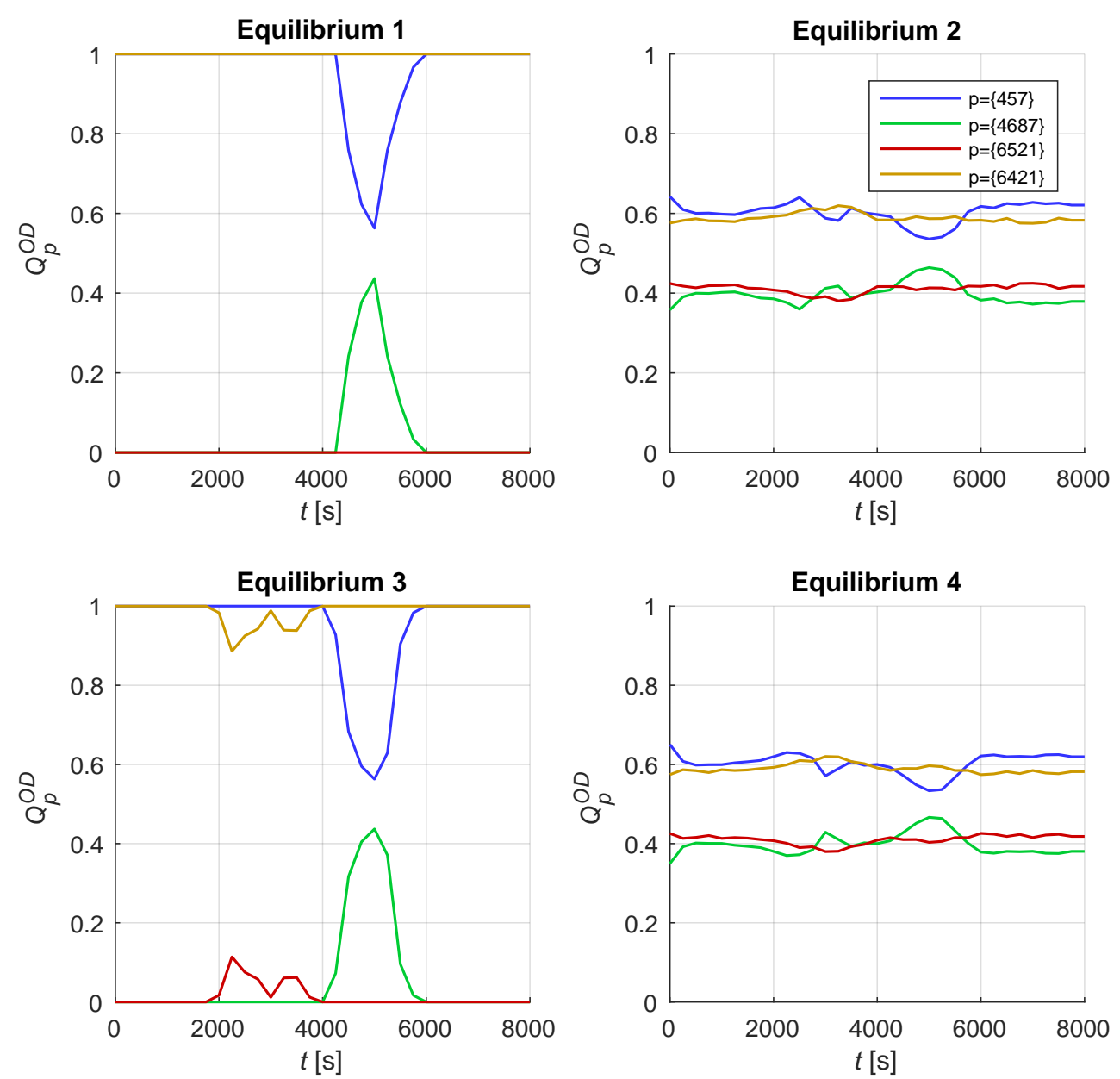

Figure 17 Evolution of regional path flows $Q_{p}^{O D}$ as function of the simulation time $\mathbf{t}$ [s], for all four regional paths. Each subplot corresponds to each of the four network equilibrium.

larger period of time and reduces the mean speed $v(t)$. The changes in the traffic dynamics induce changes in the evolution of the mean speed profile in the regions and consequently on $\sigma_{v}$.

We start by analyzing the modeled traffic states in the regions for Equilibrium 1. We note that for this demand scenario 1, none of the four regional paths cross region 3. This region is then maintained at the free-flow speed during all the simulation. Between 0 and 1000 seconds, all drivers choose regional paths $p=\{6421\}$ and $p=\{457\}$ as $U_{p=\{6421\}}^{61}<U_{p=\{6521\}}^{61}$ and $U_{p=\{457\}}^{47}<U_{p=\{4687\}}^{47}$. The vehicles accumulation increases in all regions except in region 3 and the mean speed $v(t)$ decreases as observed in Fig. 16. There are two demand peaks in the period between 1000 and 6000 seconds (see Fig. 15 (a)). Initially, between 1000 and 4000 seconds, all drivers choose the same regional paths (i.e. $p=\{6421\}$ and $p=\{457\}$ ) as their travel times are still the minimal ones. The increase of the demand traveling on OD 61 leads to an increase of the vehicles accumulation 
traveling on regional path $p=\{6421\}$ between 1000 and 2750 seconds, reducing the mean speed $v(t)$ in these four regions. Between 2750 and 4000 seconds, the vehicles accumulation decreases and the mean speed $v(t)$ increases in the same regions. Between 4000 and 4250 seconds, the number of vehicles traveling on regional path $p=\{6421\}$ decreases while it increases on regional path $p=\{457\}$. This leads to different evolution trends of the vehicles accumulation in regions 5 and 6 . There is an increase of the vehicles accumulation in region 5, leading to a reduction of the mean speed $v(t)$. In region 6 , we observe an inverse trend. At $t=4250$ seconds, drivers start to switch from regional path $p=\{457\}$ to $p=\{4687\}$. In order to better understand this switch, we analyze the utilities (see Eq. 6) of the two regional paths of OD 47:

$$
\begin{gathered}
U_{p=\{457\}}^{47}=\frac{246}{\bar{v}_{4}}+\frac{464}{\bar{v}_{5}}+\frac{387}{\bar{v}_{7}} \\
U_{p=\{4687\}}^{47}=\frac{234}{\bar{v}_{4}}+\frac{414}{\bar{v}_{6}}+\frac{464}{\bar{v}_{8}}+\frac{130}{\bar{v}_{7}}
\end{gathered}
$$

where the average trip length values are gathered from Table 2 and the mean speed values from the inspection of Fig. 16.

We recall that drivers aim to minimize their own regional path travel times, i.e. they are utility minimizers. Under User Equilibrium conditions, between 4250 and 6000 seconds, we have that $U_{p=\{457\}}^{47} \approx U_{p=\{4687\}}^{47}$. The vehicles accumulation increases in region 5 , reducing its mean speed $v(t)$. This leads to an increase of the travel time of regional path $p=\{457\}$, i.e. increasing Eq. 19. On the other hand, during the period between 4000 and 4250 seconds, there are no vehicles traveling on regional path $p=\{4687\}$, keeping region 8 still at the free-flow speed. Moreover, the average travel distance of regional path $p=\{4687\}$ in region 7 is 130 meters, which is much lower than the 390 meters for regional path $p=\{457\}$. The travel time that is added by region 8 to Eq. 20 is compensated by the lower travel time in region 7 . This is because of the smaller average travel distance for regional path $p=\{4687\}$ in region 7 in comparison with $p=\{457\}$. To maintain the User Equilibrium conditions, the fraction of drivers switching to regional path $p=\{4687\}$ increases as region 5 becomes more congested, i.e. during the period between 4000 and 5000 seconds. After 5000 seconds, the number of vehicles traveling on these two regional paths decreases as the demand traveling on OD 47 also decreases. The vehicles accumulation on region 5 decreases and its mean speed $v(t)$ increases, reducing the travel time of regional path $p=\{457\}$. Then, there are more drivers choosing this regional path such that the User Equilibrium conditions are satisfied. After 6000 seconds, the demand levels on both OD pairs are constant and equal to $0.1 \mathrm{veh} / \mathrm{s}$. The vehicles accumulation in the regions are maintained at a constant level as well as their mean speeds $v(t)$ (see Fig. 16). All drivers choose regional paths $p=\{6421\}$ and $p=\{457\}$ as their travel times 
are perceived as the minimal ones. In this case, the User Equilibrium conditions correspond again to $U_{p=\{6421\}}^{61}<U_{p=\{6521\}}^{61}$ and $U_{p=\{457\}}^{47}<U_{p=\{4687\}}^{47}$, similar to the initial period between 0 and 1000 seconds.

We now investigate the influence of $\sigma_{v}$ on the regional path flows at equilibrium. For this, we analyze the differences in the evolution trends of the mean speed $v(t)$ in the regions as well as the differences of the regional path flows between Equilibrium 3 and 1. We start by stressing out the fact that as $\sigma_{v} \rightarrow 0 \Longrightarrow v_{r} \rightarrow \overline{v_{r}}$, Eq. 8 is reduced to:

$$
U_{p}^{O D} \rightarrow \sum_{r \in X}\left(\frac{\bar{L}_{r p}}{\bar{v}_{r}}\right) \delta_{r p}, \forall p \in \Omega^{O D} \wedge \forall(O, D) \in W
$$

This formulation of the regional path utility $U_{p}^{O D}$ is similar to the one defined in Eq. 6 for Equilibrium 1. The second term $\frac{\bar{L}_{r p} v_{r}}{\bar{v}_{r}^{2}}$ in Eq. 8 only plays a significant role in the drivers' perception of travel times for larger values of $\sigma_{v}$. This happens during the charging and discharging periods of the regions, i.e. between 1000 and 6000 seconds. The first demand peak occurs between 1000 and 4500 seconds on OD 61. During this period and in the case of Equilibrium 1, all drivers choose regional path $p=\{6421\}$. In the case of Equilibrium 3, the travel time of regional path $p=\{6521\}$ is perceived as the minimal one for an increasing number of Monte Carlo samples, as the demand peak increases until 2750 seconds. After this time instant, the demand decreases and the travel time of regional path $p=\{6421\}$ is perceived as the minimal one for a larger number of Monte Carlo samples. The fraction of drivers choosing regional path $p=\{6521\}$ increases during the charging period and decreases during the discharging period of the regions. A similar trend is observed between 4000 and 6000 seconds for the second demand peak of OD 47. The travel time of regional path $p=\{4687\}$ is perceived as the minimal one for an increasing number of Monte Carlo samples as the demand peak increases until 5000 seconds. Therefore, the fraction of drivers choosing this regional path increases.

We now investigate the influence of $\sigma_{L}$ on the regional path flows at equilibrium. For this purpose, we analyze the differences between the evolution trends of the mean speed $v(t)$ in the regions as well as the differences of the regional path flows between Equilibrium 2 and 1 . We note that as $\sigma_{L} \rightarrow 0 \Longrightarrow L_{r p} \rightarrow \bar{L}_{r p}$. The utility of regional path $p$ defined in Eq. 7 is reduced to the one set up for Equilibrium 1 in Eq. 6. Batista, Leclercq, and Geroliminis (2019) shows that the standard deviation $\sigma_{L}$ of the trip length distributions depends on the topology of the city network in connection with the definition of the regions, i.e. the partition of the city network. The authors show that for Origin and Destination regions of a regional path, the shape of the trip length distributions depend on the spatial distribution of the origin and destination nodes inside these regions as well as on the border nodes that allow to travel to the adjacent regions. The trip length distributions 
for intermediate regions depend on the topology of the city network as well as on the incoming and outgoing links directions of the border nodes. For example, the standard deviation of the trip length distribution of regional path $p=\{4687\}$ inside region 6 is 0 . This happens because the test network represents an area of the Lyon city, where there are a lot of one-way streets. The definition of the city network partitioning depicted in Fig. 14 (a) sets only one border node that allows regions 4 to 6 and then from 6 to 8 . Therefore, there is only one shortest-path between these two border nodes and the travel distance inside region 6 is always equal to 414 meters. Fig. 18 depicts the distributions of the standard deviations $\sigma_{L}$ for the total 205 internal and regional paths calculated for the $6^{\text {th }}$ district Lyon network. We distinguish these distributions between the internal paths and the Origin, Intermediate and Destination regions for the regional paths. We observe that $\sigma_{L}$ is in general greater than 0 . Even though we observe that $\sim 30 \%$ of the intermediate regions have a small $\sigma_{L}$ value, this value can go up to $\sigma_{L} \sim 150-200$ meters. Generally, we observe that $\sigma_{L}>>0$ meters, giving $\sigma_{L}$ a significant role in the calculation of the regional path flows at equilibrium. Fig. 17 depicts the differences between the regional path flows for Equilibrium 2 and 1. For Equilibrium 1, all drivers choose regional paths $p=\{6421\}$ and $p=\{457\}$, except between 4000 and 6000 seconds. During the latter period, drivers choose between regional paths $p=\{457\}$ and $p=\{4687\}$, such that $U_{p=\{457\}}^{47} \approx U_{p=\{4687\}}^{47}$ as previously discussed. For Equilibrium 2, around $\sim 60 \%$ of the drivers choose regional paths $p=\{457\}$ and $p=\{6421\}$, while $\sim 40 \%$ choose the regional paths $p=\{4687\}$ and $p=\{6521\}$. The different drivers' regional paths choices between Equilibrium 1 and 2 induce significant changes in the traffic dynamics in the regions (see Fig. 16).

We now investigate the combined influence of $\sigma_{L}$ and $\sigma_{v}$ on the drivers choices for their regional paths. For this purpose, we analyze the differences between the evolution trends of the mean speed $v(t)$ in the regions as well as the differences of the regional path flows between Equilibrium 4 and 3. We stress out that when $\sigma_{L}>>\sigma_{v}$, the regional path utility $U_{p}^{O D}$ defined in Eq. 9 reduces to:

$$
U_{p}^{O D} \approx \sum_{r \in X}\left(\frac{\bar{L}_{r p}}{\bar{v}_{r}}+\frac{L_{r p}}{\bar{v}_{r}}\right) \delta_{r p}, \forall p \in \Omega^{O D} \wedge \forall(O, D) \in W
$$

where only the trip length distributions $L_{r p}$ influence the regional paths utilities $U_{p}^{O D}$ and consequently the drivers choices. When $\sigma_{L}$ is dominant over $\sigma_{v}$, the regional path flows between Equilibrium 2 and 4 will be close, as verified in Fig. 17. This also leads to similar traffic dynamics in the regions between Equilibrium 2 and 4 (see Fig. 16). Nevertheless, $\sigma_{L}$ is not always dominant over $\sigma_{v}$ and the previous result cannot be generalized. In more congested and more complex demand scenarios, where there are more interactions between different regional paths crossing the same region, the $\sigma_{v}$ becomes more significant and plays an important role in the drivers choices for their regional paths. 

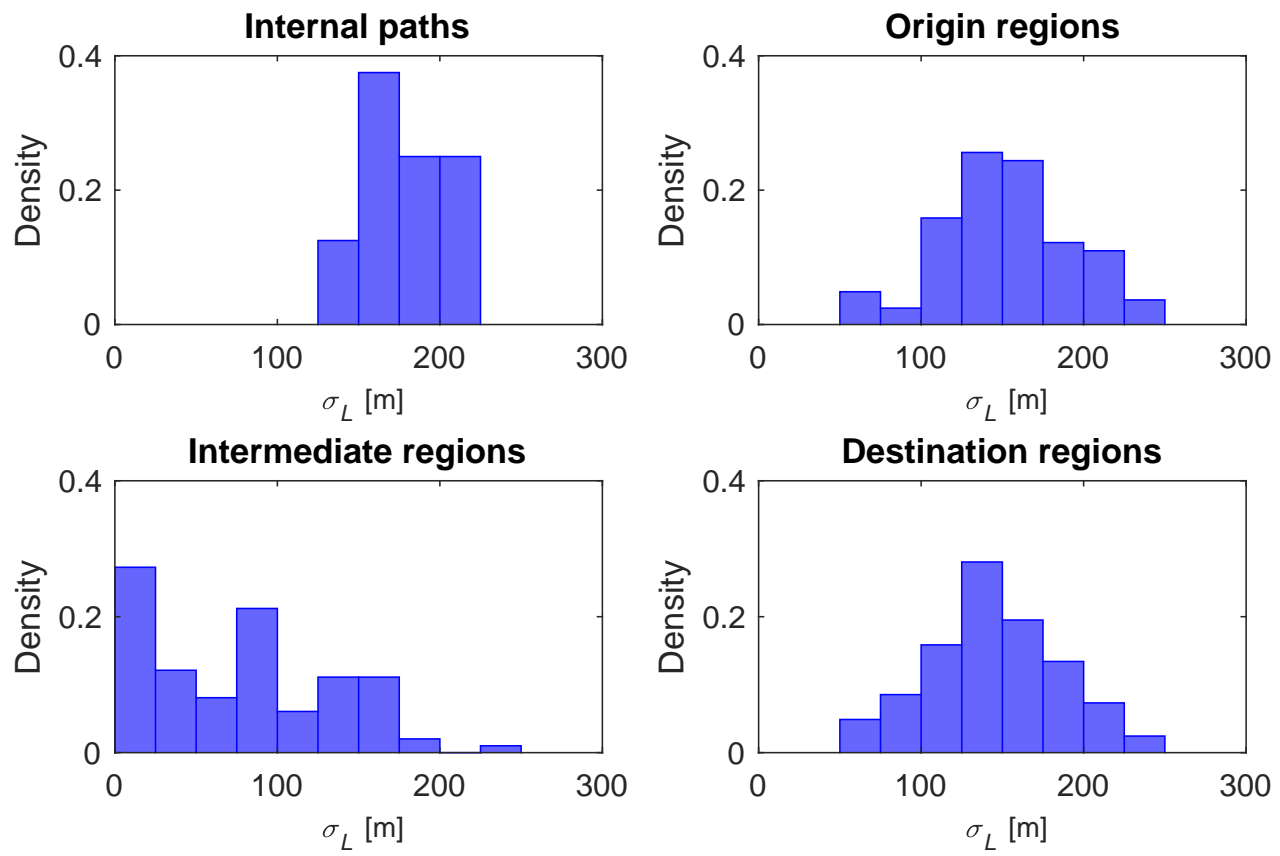

Figure 18 Distributions of $\sigma_{L}$ for the internal paths as well as for the Origin, Intermediate and Destination regions of all 205 regional paths obtained for the $6^{\text {th }}$ district Lyon network. The binwidth is set to 25 meters.

\subsection{Analysis of the combined influence of $\sigma_{L}$ and $\sigma_{v}$ on the network equilibrium}

In this section, we investigate the combined influence of $\sigma_{L}$ and $\sigma_{v}$ on the drivers choices for their regional paths, for the demand scenario 2 introduced in Sect. 4.1. We can distinguish two important time instants of this demand scenario. The first moment lies between 1000 and 2000 seconds where there is a demand peak on OD 32 and a sudden increase of the demand traveling on OD 16. The second moment occurs between 4000 and 6000 seconds, that coincides with the sudden decrease of the demand on OD 16 and the three demand peaks on OD 48, 74 and 57. Fig. 19 depicts the evolution of the traffic dynamics in the regions for this demand scenario 2. One can observe that there are not significant differences between the traffic dynamics for Equilibrium 2 and 4, between 1000 and 2000 seconds. This happens because $\sigma_{L}$ is dominant over $\sigma_{v}$, i.e. $\sigma_{L}>>\sigma_{v}$, similar to the case that we have dissected in the previous section. During the second period between 4000 and 6000 seconds, the demand peaks lead region 5 to become more congested and the congestion propagates to the adjacent regions 4,6 and 7. This yields significant changes in the traffic dynamics in these regions and therefore on the mean speed $v(t)$, during the assignment period of 250 seconds for which the network equilibria are calcualted. In this case, $\sigma_{v}$ becomes more significant and also plays an important role in the drivers choices for their regional path choices. The influence of $\sigma_{v}$ on the drivers' choices for their regional paths leads to significative differences between the evolution 

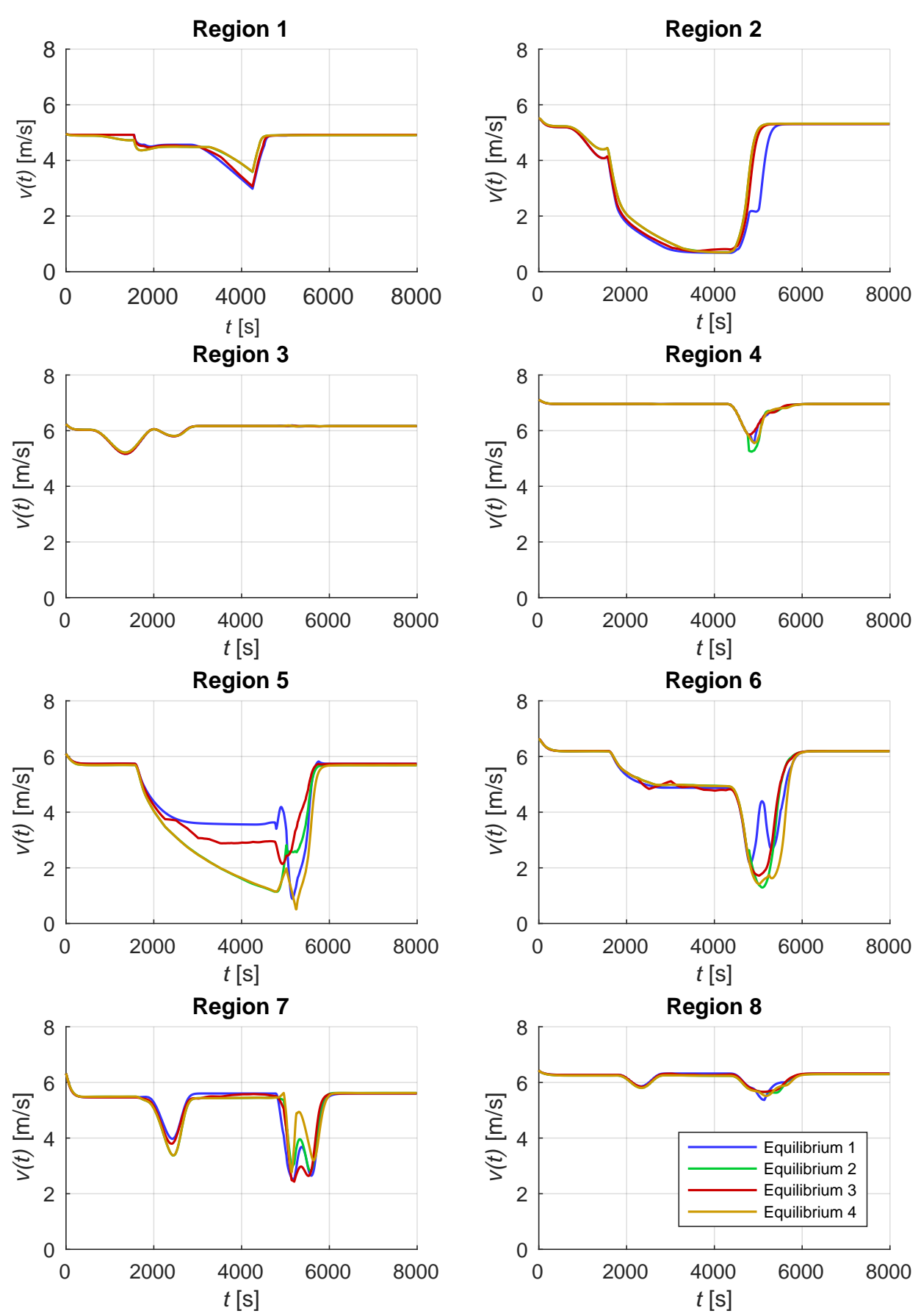

Figure 19 Same as in Fig. 17, but for the demand scenario 2. 
trends of the traffic dynamics in regions 4, 5, 6 and 7 between 4000 and 6000 seconds as depicted in Fig. 19.

\section{Conclusions}

In this paper, we propose a regional dynamic traffic assignment framework for MFD-based models that explicitly account for trip length distributions $L_{r p}$ and the evolution of the regional mean speed $v_{r}\left(n_{r}\right)$. We set four formulations of the network equilibrium, based on which terms are considered distributed. We show that Equilibrium 4, which considers that both trip lengths and regional mean speed are distributed, should be preferred. The results shown in this paper confirm that we cannot neglect the variability of trip lengths inside the regions. In addition, we showed the importance of considering that the regional mean speed is also distributed, in order to account for the correlation between regional paths. The proposed regional dynamic traffic assignment in the MFD context is an extension of the framework discussed by Yildirimoglu and Geroliminis (2014). First, our framework explicitly accounts for multiple trip lengths inside the regions that are explicitly calculated. Second, we do not assume any prior statistical distribution for the regional path travel times, whereas Yildirimoglu and Geroliminis (2014) considered that they are independent and identically distributed variables. Moreover, the authors used the MNL formulation to calculate the network equilibrium. Instead, we considered the distributions of trip lengths and regional mean speed and used Monte Carlo simulations to calculate the network equilibrium. Contrary to the MNL formulation, our framework was able to capture the correlation between regional paths. We also analyzed the implementation of our framework on the $6^{\text {th }}$ district of the Lyon network, where the trip lengths are explicitly calculated following the specific regional path, as proposed in Batista et al. (2018) and Batista, Leclercq, and Geroliminis (2019). Since we calculated the network equilibrium for small simulation periods, the mean speed did not vary greatly and $\sigma_{v}$ was low. In this case, $\sigma_{L}$ dominated in the calculation of the utility functions and was responsible for the changes in the system dynamics observed. However, when there is an important interaction between demand peaks of different OD pairs, $\sigma_{v}$ becomes more significant and also plays an important role in the drivers choices for their regional paths and on the modeled traffic dynamics in the regions.

In future research, we plan to investigate the time-dependence of trip lengths, i.e. their dependence on the traffic dynamics in the regions, and propose a robust and computationally light methodology to update the trip length distributions accordingly. This will allow to account for the variation of the trip length distributions variances in the calculation of the regional network equilibrium. We also plan to extend this methodological framework to account for different kinds of drivers' behavior, such as bounded rational (Mahmassani and Chang 1987, Di and Liu 2016, Batista, Zhao, and Leclercq 2018) and regret-aversion (Chorus 2014, Li and Huang 2016), as well as heterogeneous preferences for more reliable travel times. 
Batista and Leclercq: Regional dynamic traffic assignment framework for MFD multi-regions models

\section{Acknowledgments}

We thank the anonymous reviewers for their comments and suggestions that have improved this manuscript.

This project is supported by the European Research Council (ERC) under the European Union's Horizon 2020 research and innovation program (grant agreement No 646592 - MAGnUM project). S. F. A. Batista also acknowledges funding support by the region Auvergne-Rhône-Alpes (ARC7 Research Program).

\section{References}

Ambühl L, Loder A, Zheng N, Axhausen KW, Menendez M, 2019 Approximative network partitioning for mfds from stationary sensor data. Transportation Research Record in press.

Ambühl L, Menendez M, 2016 Data fusion algorithm for macroscopic fundamental diagram estimation. Transportation Research Part C: Emerging Technologies 71:184-197, URL http://dx.doi.org/10. $1016 / j \cdot \operatorname{trc} .2016 .07 .013$.

Arnott R, 2013 A bathtub model of downtown traffic congestion. Journal of Urban Economics 76:110-121, URL http://dx.doi.org/10.1016/j.jue.2013.01.001.

Azevedo J, Santos Costa M, Silvestre Madeira J, Vieira Martins E, 1993 An algorithm for the ranking of shortest paths. European Journal of Operational Research 69:97-106, URL http://dx.doi.org/10. 1016/0377-2217 (93) 90095-5.

Batista SFA, Leclercq L, 2018 Introduction of multi-regional mfd-based models with route choices: the definition of regional paths. PLURIS 2018 - $8^{\text {th }}$ LUSO-BRAZILIAN CONGRESS for Urban, Regional, Integrated and Sustainable Planning (Coimbra, Portugal), URL https://www.dec.uc.pt/pluris2018/ Paper1356.pdf.

Batista SFA, Leclercq L, Geroliminis N, 2019 Estimation of regional trip length distributions for the calibration of the aggregated network traffic models. Transportation Research Part B: Methodological 122:192217, URL http://dx.doi.org/10.1016/j.trb.2019.02.009.

Batista SFA, Leclercq L, Krug J, Geroliminis N, 2018 Trip length estimation for the macroscopic traffic simulation: scaling microscopic into macroscopic networks. 97th Annual Meeting Transportation Research Board (Washington DC, USA).

Batista SFA, Zhao CL, Leclercq L, 2018 Effects of users bounded rationality on a traffic network performance: A simulation study. Journal of Advanced Transportation 2018:20, URL http://dx.doi.org/10.1155/ 2018/9876598.

Bekhor S, Ben-Akiva ME, Ramming S, 2002 Adaptation of logit kernel to route choice situation. Transportation Research Record 1805:78-85, URL http://dx.doi.org/10.3141/1805-10.

Bekhor S, Prashker JN, 2001 Stochastic user equilibrium formulation for the generalized nested logit model. Transportation Research Record 1752:84-90, URL http://dx.doi.org/10.3141/1752-12.

Ben-Akiva M, Bergman MJ, Daly A, Ramaswamy V, 1984 Modeling interurban route choice behaviour. Proceedings of the 9th International Symposium on Transportation and Traffic Theory (Utrecht, The Netherlands). 
Ben-Akiva M, Bierlaire M, 1999 Handbook of Transportation Science, chapter Discrete Choice Methods and their Applications to Short Term Travel Decisions, 5-33 (Boston, MA: Springer US), ISBN 978-1-46155203-1, URL http://dx.doi.org/10.1007/978-1-4615-5203-1_2.

Ben-Akiva M, Gao WZ S, Wen Y, 2012 A dynamic traffic assignment model for highly congested urban networks. Transportation Research Part C: Emerging Technologies 24:62-82, URL http://dx.doi. org $/ 10.1016 / j . \operatorname{trc} .2012 .02 .006$.

Bierlaire M, Frejinger E, 2005 Route choice models with subpath components. Proceedings of the $5^{\text {th }}$ Swiss Transport Research Conference (Ascona, Switzerland).

Bliemer MCJ, Bovy PHL, Li H, 2007 Some properties and implications of stochastically generated route choice sets. Proceedings of the 6th Tristan Conference (Pukhet, Thailand).

Bolduc D, 1999 A pratical technique to estimate multinomial probit models in transportation. Transportation Research Part B 33:63-79, URL http://dx.doi.org/10.1016/S0191-2615(98)00028-9.

Bovy PHL, Bekhor S, Prato CG, 2008 The factor of revised path size: an alternative derivation. Transportation Research Record 2076:132-140, URL http://dx.doi.org/10.3141/2076-15.

Casadei G, Bertrand V, Gouin B, Canudas-de-Wit C, 2018 Aggregation and travel time calculation over large scale traffic networks: An empiric study on the grenoble city. Transportation Research Part C: Emerging Technologies 95:713-730, URL http://dx.doi.org/10.1016/j.trc.2018.07.033.

Cascetta E, 2001 Transportation Systems Engineerin: Theory and Methods (Kluwer Academic Publishers), ISBN 978-1-4757-6873-2, URL http://dx.doi.org/10.1007/978-1-4757-6873-2.

Cascetta E, Nuzzolo A, Russo F, Vitetta A, 1996 A modified logit route choice model overcoming path overlapping problems: specification and some calibration results for interurban networks. Proceedings of the $13^{\text {th }}$ International Symposium on Transportation and Traffic Theory, 697-711 (Lyon, France).

Chen A, Pravinvongvuth S, Xu X, Ryu S, Chootinan P, 2012 Examining the scaling effect and overlapping problem in logit-based stochastic user equilibrium models. Transportation Research Part A 46:1343-1358, URL http://dx.doi.org/10.1016/j.tra.2012.04.003.

Chorus C, 2014 A generalized random regret minimization model. Transportation Research Part B: Methodological 68:224-238, URL http://dx.doi.org/10.1016/j.trb.2014.06.009.

Chu C, 1989 A paired combinatorial logit model for travel demand analysis. Proceedings of the $5^{\text {th }}$ World Conference on Transportation Research, Ventura, 295-309 (Ventura, USA).

Corthout R, Flötteröd G, Viti F, Tampre CMJ, 2012 Non-unique flows in macroscopic first-order intersection models. Transportation Research Part B: Methodological 46:343-359, URL http://dx.doi.org/10. $1016 / j \cdot \operatorname{trb} .2011 \cdot 10 \cdot 011$.

Daganzo C, 2007 Urban gridlock: Macroscopic modeling and mitigation approaches. Transportation Research Part B: Methodological 41:49-62, URL http://dx.doi.org/10.1016/j.trb.2006.03.001. 
Batista and Leclercq: Regional dynamic traffic assignment framework for MFD multi-regions models

Daganzo C, Sheffi Y, 1977 On stochastic models of traffic assignment. Transportation Science 11:253-274, URL http://dx.doi.org/10.1287/trsc.11.3.253.

Daganzo CF, 1982 Unconstrained extremal formulation of some transportation equilibrium problems. Transportation Science (16):332-360, URL http://dx.doi.org/10.1287/trsc.16.3.332.

de la Barra T, Perez B, Anez J, 1993 Multidimensional path search and assignment. Proceedings of the 21 ${ }^{\text {st }}$ PTRC Summer Annual Meeting (Manchester, England).

Di X, He X, Guo X, Liu HX, 2014 Braess paradox under the boundedly rational user equilibria. Transportation Research Part B 67:86-108, URL http://dx.doi.org/10.1016/j.trb.2014.04.005.

Di X, Liu H, Pang J, Ban X, 2013 Boundedly rational user equilibria (brue): mathematical formulation and solution sets. Transportation Research Part B 57:300-313, URL http://dx.doi.org/10.1016/j.trb. 2013.06 .008 .

Di X, Liu HX, 2016 Boundedly rational route choice behavior: A review of models and methodologies. Transportation Research Part B: Methodological 85:142-179, URL http://dx.doi.org/10.1016/j.trb. 2016.01 .002$.

Dial R, 1971 A probabilistic multipath traffic assignment model which obviates path enumeration. Transportation Research (5):83-113, URL http://dx.doi.org/10.1016/0041-1647(71)90012-8.

Flötteröd G, Bierlaire, 2013 Metropolis-hastings sampling of paths. Transportation Research Part B 48:53-66, URL http://dx.doi.org/10.1016/j.trb.2012.11.002.

Fosgerau M, 2015 Congestion in the bathtub. Economics of Transportation 4:241-255, URL http://dx.doi. org/10.1016/j.ecotra.2015.08.001.

Frejinger E, Bierlaire M, 2007 Capturing correlation with subnetworks in route choice models. Transportation Research Part B 41(3):363-378, URL http://dx.doi.org/10.1016/j.trb.2006.06.003.

Frejinger E, Bierlaire M, Ben-Akiva M, 2009 Sampling of alternatives for route choice modelling. Transportation Research Part B 43:984-994, URL http://dx.doi.org/10.1016/j.trb.2009.03.001.

Geroliminis N, Daganzo C, 2008 Existence of urban-scale macroscopic fundamental diagrams: Some experimental findings. Transportation Research Part B: Methodological 42:759-770, URL http://dx.doi . org/10.1016/j.trb.2008.02.002.

Geroliminis N, Sun J, 2011 Hysteresis phenomena of a macroscopic fundamental diagram in freeway networks. Procedia - Social and Behavioral Sciences 17:213-228, URL http://dx.doi.org/10.1016/j.sbspro. 2011.04 .515$.

Godfrey JW, 1969 The mechanism of a road network. Traffic Engineering and Control 11:323-327, URL https://trid.trb.org/view.aspx?id=117139.

Herman R, Prigogine I, 1979 A two-fluid approach to town traffic. Science 204:148-151, URL http://dx. doi.org/10.1126/science.204.4389.148. 
Iryo T, 2011 Multiple equilibria in a dynamic traffic network. Transportation Research Part B: Methodological 45:867-879, URL http://dx.doi.org/10.1016/j.trb.2011.02.010.

Lamotte R, Geroliminis N, 2016 The morning commute in urban areas: Insights from theory and simulation. Transportation Research Board 95 ${ }^{\text {th }}$ Annual Meeting., 16-2003.

Laval J, Leclercq L, Chiabaut N, 2017 Minimal parameter formulations of the dynamic user equilibrium using macroscopic urban models: Freeway vs city streets revisited. Transportation Research Part B: Methodological in press, URL http://dx.doi.org/10.1016/j.trb.2017.08.027.

Leclercq L, 2007 Hybrid approaches to the solutions of the "lighthill-whitham-richards" model. Transportation Research Part B: Methodological 41:701-709, URL http://dx.doi.org/10.1016/j.trb.2006. 11.004.

Leclercq L, Geroliminis N, 2013 Estimating mfds in simple networks with route choice. Transportation Research Part B: Methodological 57:468 - 484, URL http://dx.doi.org/https://doi .org/10.1016/ j.trb. 2013.05 .005$.

Leclercq L, Sénécat A, Mariotte G, 2017 Dynamic macroscopic simulation of on-street parking search: A trip-based approach. Transportation Research Part B: Methodological 101:268-282, URL http://dx . doi.org/10.1016/j.trb.2017.04.004.

Li M, Huang HJ, 2016 A regret theory-based route choice model. Transportmetrica A: Transportation Science 13:250-272, URL http://dx.doi.org/10.1080/23249935.2016.1252445.

Loder A, Ambühl L, Menendez M, Axhausen KW, 2017 Empirics of multi-modal traffic networks using the 3d macroscopic fundamental diagram. Transportation Research Part C: Emerging Technologies 82:88-101, URL http://dx.doi.org/10.1016/j.trc.2017.06.009.

Lopez C, Leclercq L, Krishnakumari P, Chiabaut N, van Lint H, 2017 Revealing the day-to-day regularity of urban congestion patterns with $3 d$ speed maps. Scientific Reports 7:1-11, URL http://dx.doi.org/ $10.1038 / \mathrm{s} 41598-017-14237-8$.

Lu CC, Mahmassani HS, Zhou X, 2008 A bi-criterion dynamic user equilibrium traffic assignment model and solution algorithm for evaluating dynamic road pricing strategies. Transportation Research Part C: Emerging Technologies 16(4):371 - 389, URL http://dx.doi.org/10.1016/j.trc.2007.08.002.

Mahmassani H, Saberi M, Zockaie A, 2013 Urban network gridlock: Theory, characteristics, and dynamics. Transportation Research Part C: Emerging Technologies 36:480-497, URL http://dx.doi.org/10. $1016 / j . \operatorname{trc} .2013 .07 .002$.

Mahmassani H, Williams JC, Herman R, 1984 Investigation of network-level traffic flow relationships: Some simulation results. Transportation Research Record: Journal of the Transportation Research Board 971:121-130, URL http://dx.doi.org/10.3141/2315-16.

Mahmassani HS, Chang GL, 1987 On boundedly rational user equilibrium in transportation systems. Transportation Science 21:89-99, URL http://dx.doi.org/10.1287/trsc.21.2.89. 
Mariotte G, Leclercq L, 2019 Flow exchanges in multi-reservoir systems with spillbacks. Transportation Research Part B: Methodological 122:327 - 349, URL http://dx.doi.org/10.1016/j.trb.2019.02. 014.

Mariotte G, Leclercq L, Laval JA, 2017 Macroscopic urban dynamics: Analytical and numerical comparisons of existing models. Transportation Research Part B 101:245-267, URL http://dx.doi.org/10.1016/ j.trb.2017.04.002.

McFadden D, 1978 Spatial Interaction Theory and Planning Models, chapter Modelling the choice of residential location, 75-96 (Cambridge, MA: MIT Press).

Merchant DK, Nemhauser GL, 1978a A model and an algorithm for the dynamic traffic assignment problems. Transportation Science 12(3):183-199, URL http://dx.doi.org/10.1287/trsc.12.3.183.

Merchant DK, Nemhauser GL, 1978b Optimality conditions for a dynamic traffic assignment model. Transportation Science 12(3):200-207, URL http://dx.doi.org/10.1287/trsc.12.3.200.

Nie YM, Zhang HM, 2010 Solving the dynamic user optimal assignment problem considering queue spillback. Networks and Spatial Economics 10:49-71, URL http://dx.doi.org/10.1007/s11067-007-9022-y.

Nielsen OA, 2000 A stochastic transit assignment model considering differences in passengers utility functions. Transportation Research Part B 34(5):377-402, URL http://dx.doi.org/10.1016/S0191-2615(99) 00029-6.

Nielsen OA, Daly A, Frederiksen RD, 2002 A stochastic route choice model for car travellers in the copenhagen region. Networks and Spatial Economics 2:327-346, URL http://dx.doi.org/10.1023/A:102089542.

Peeta S, Ziliaskopoulos AK, 2001 Foundations of dynamic traffic assignment: The past, the present and the future. Networks and Spatial Economics 1:233-265, URL http://dx.doi.org/10.1023/A: 1012827724856.

Prashker JN, Bekhor S, 1998 Investigation of stochastic network loading procedures. Transportation Research Record 1645:94-102, URL http://dx.doi.org/10.3141/1645-12.

Prashker JN, Bekhor S, 2000 Congestion, stochastic, and similarity effects in stochastic user equilibrium. Transportation Research Record 1733:80-87, URL http://dx.doi.org/10.3141/1733-11.

Prato CG, 2009 Route choice modelling: past, present and future research directions. Journal of Choice Modelling 2:65-100, URL http://dx.doi.org/10.1016/S1755-5345(13)70005-8.

Prato CG, Bekhor S, 2006 Applying branch and bound techniques to route choice set generation. Transportation Research Record 19-28, URL http://dx.doi.org/10.3141/1985-03.

Ramming M, 2002 Network Knowledge and Route Choice, PhD thesis (Massachusetts Institute of Technology).

Saeedmanesh M, Geroliminis N, 2016 Clustering of heterogeneous networks with directional flows based on "snake" similarities. Transportation Research Part B: Methodological 91:250-269, URL http://dx. doi.org/10.1016/j.trb.2016.05.008. 
Saeedmanesh M, Geroliminis N, 2017 Dynamic clustering and propagation of congestion in heterogeneously congested urban traffic networks. Transportation Research Procedia 23:962-979, URL http://dx.doi . org $/ 10.1016 / j \cdot \operatorname{trb} .2017 .08 .021$.

Sbayti H, Lu CC, Mahmassani HS, 2007 Efficient implementation of method of successive averages in simulation-based dynamic traffic assignment models for large-scale network applications. Transportation Research Record: Journal of the Transportation Research Board 2029:22-30, URL http://dx. doi.org/10.3141/2029-03.

Shafiei S, Gu Z, Saberi M, 2018 Calibration and validation of a simulation-based dynamic traffic assignment model for a large-scale congested network. Simulation Modelling Practice and Theory 86:169-186, URL http://dx.doi.org/10.1016/j.simpat.2018.04.006.

Sheffi Y, 1985 Urban Transportation networks: Equilibrium Analysis with Mathematical Programming Methods, chapter 10 and 11 (United States of America: Prentice Hall Inc.).

Simon HA, 1957 A behavioral model of rational choice (New York: Wiley).

Simon HA, 1966 Theories of Decision-Making in Economics and Behavioural Science, 1-28 (London: Palgrave Macmillan UK), ISBN 978-1-349-00210-8, URL http://dx.doi.org/10.1007/978-1-349-00210-8_ 1.

van der Zijpp NJ, Catalano SF, 2005 Path enumeration by finding the constrained k-shortest paths. Transportation Research Part B: Methodological 39:545-563, URL http://dx.doi.org/10.1016/j.trb. 2004.07 .004$.

Viti F, Tampère CMJ, 2010 New developments in transport planning: Advances in dynamic traffic assignment (Cheltenham UK: Edward Elgar), ISBN 9781848449633, URL http://dx.doi.org/10.4337/ 9781781000809.00007.

Vovsha P, 1997 The cross-nested logit model: an application to mode choice in the tel aviv metropolitan area. Transportation Research Record 1607:13-20, URL http://dx.doi.org/10.3141/1607-02.

Wardrop JG, 1952 Some theoretical aspects of road traffic research. Institution of Civil Engineering 1:325-362, URL http://dx.doi.org/10.1680/ipeds.1952.11259.

Wie BW, Tobin RL, Carey M, 2002 The existence, uniqueness and computation of an arc-based dynamic network user equilibrium formulation. Transportation Research Part B 36:897-918, URL http://dx. doi.org/10.1016/S0191-2615(01)00041-8.

Yildirimoglu M, Geroliminis N, 2014 Approximating dynamic equilibrium conditions with macroscopic fundamental diagrams. Transportation Research Part B: Methodological 70:186-200, URL http://dx.doi. org/10.1016/j.trb.2014.09.002.

Zhang K, Mahmassani HS, Lu CC, 2013 Dynamic pricing, heterogeneous users and perception error: Probit-based bi-criterion dynamic stochastic user equilibrium assignment. Transportation Research Part C: Emerging Technologies 27:189 - 204, URL http://dx.doi.org/10.1016/j.trc.2012.05.001, selected papers from the Seventh Triennial Symposium on Transportation Analysis (TRISTAN VII). 
Batista and Leclercq: Regional dynamic traffic assignment framework for MFD multi-regions models

Zhu S, Levinson D, 2015 Do people use the shortest path? an empirical test of wardrop's first principle. PLoS ONE (10):1-18, URL http://dx.doi.org/10.1371/journal.pone.0134322. 